\title{
Role of Gemcitabine in the Treatment of Advanced and Metastatic Breast Cancer
}

\author{
Volker Heinemann \\ Medical Clinic III, Klinikum Grosshadern, Munich, Germany
}

\author{
Key Words \\ Breast cancer · Combination therapy · Gemcitabine • \\ Single agent
}

\begin{abstract}
Gemcitabine is an antimetabolite drug with proven antitumor activity and tolerability in metastatic breast cancer. In a total of nine studies, gemcitabine monotherapy has reached response rates of up to $37 \%$ in the first-line setting, $26 \%$ in the second-line setting, and $18 \%$ or better in the third-line setting. Gemcitabine is an excellent choice for combination therapy by its unique mechanism of action and favorable toxicity profile, thus limiting the risk of pretreatment-related drug resistance and overlapping toxicity, and by its potential for synergistic interaction with some combination partners as indicated in preclinical studies. Numerous phase II clinical studies have combined gemcitabine with other active agents such as the taxanes, vinorelbine, vindesine, cisplatin, 5-fluorouracil, as well as anthracyclines across various regimens and conditions of pretreatment. Most of these two-drug combinations have consistently demonstrated higher efficacy than either single agent, particularly in pretreated patients. Even higher efficacy has been obtained with triple-drug regimens including gemcitabine, anthracyclines (epirubicin or doxorubicin), and paclitaxel; these regimens have yielded overall response rates of $58-92 \%$
\end{abstract}

as first-line treatment. In view of these results, gemcitabine may be regarded as a valuable alternative to the palliative treatment of metastatic breast cancer, and an excellent option for the development of effective combination treatment not only in first-line therapy, but also for intensively pretreated patients previously exposed to anthracyclines and/or the taxanes.

Copyright $\odot 2003$ S. Karger AG, Basel

\section{Introduction}

Metastatic breast cancer treatment is characterized by the availability of multiple treatment options made possible by active single agents and their combinations, the effectiveness of which is not limited to first- and secondline settings.

Single-agent therapy in chemonaive patients has achieved overall response rates of $25-55 \%$, which may be improved further to $35-80 \%$ with combination therapy [1]. Median durations of response have been in the range of 6-12 months in most studies. In randomized phase III trials, front-line monotherapy has attained median progression-free survival times of 4-8 months and median survival times of 14-22 months [2-5]. As second-line treatment, single agents have produced response rates of $20-40 \%$ and response durations of 2-8 months [1].

\section{KARGER \\ Fax +4161306 1234 \\ E-Mail karger@karger.ch \\ www.karger.com

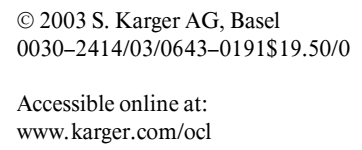

Volker Heinemann, MD, PhD

Medical Clinic III, Klinikum Grosshadern

Marchioninistrasse 15, D-81377 Munich (Germany)

Tel. +49 897095 2208, Fax +49 8970955256

E-Mail Volker.Heinemann@med3.med.uni-muenchen.de 
Despite progress in the development and understanding of new drugs, clearly defined steps toward improved survival in patients with advanced breast cancer have not been delineated recently. Strategies are confounded by the increasing exposure of patients to chemotherapy in the adjuvant setting. Nevertheless, two general strategies are apparent and should be followed: (1) improving treatment efficacy by exploring new drugs and drug combinations, and (2) ensuring that efficacy is improved with the lowest cost to quality of life.

Gemcitabine ( $2^{\prime} 2^{\prime}$-difluorodeoxycytidine, $\left.\mathrm{dFdC}\right)$ is a novel nucleoside antimetabolite that has proven systemic and preclinical antitumor activity in a variety of human solid tumors, including pancreatic, non-small-cell lung, bladder, ovarian, as well as breast tumors [6-8]. The parent compound is phosphorylated intracellularly by deoxycytidine kinase to the active metabolites, gemcitabine diphosphate, and triphosphate [9]. Gemcitabine triphosphate competes with deoxycytidine triphosphate (dCTP) for incorporation into DNA as a fraudulent base, which results in masked chain termination and inhibition of further DNA synthesis. Gemcitabine diphosphate inhibits ribonucleotide reductase, an enzyme responsible for catalyzing dCTP. The subsequent decrease in dCTP potentiates the incorporation of gemcitabine triphosphate into DNA, and enhances phosphorylation of gemcitabine since there is less dCTP to inhibit deoxycytidine kinase $[10,11]$. These self-potentiating mechanisms prolong the retention of active gemcitabine in tumor cells.

Among the multitude of available agents, gemcitabine stands out because of its good tolerability and side-effect profile [12]. The mild toxicity of gemcitabine certainly favors its combination with other antitumor drugs. In addition, the novel mechanism of action of this agent [11] reduces the possibility of pretreatment-associated drug resistance.

While gemcitabine has already demonstrated clinical efficacy as a single agent in breast cancer [13-21], knowledge regarding its treatment profile in combination therapy against this tumor is only recently emerging. On this basis, this review has evaluated the clinical applicability and efficacy of gemcitabine-based combination therapy in metastatic breast cancer.

\section{Methods}

The MEDLINE database was searched for publications related to the use of gemcitabine in breast cancer [22]. In addition to full publications, abstracts presented at the meetings of the American Society of Clinical Oncology (ASCO), the European Cancer Conference
(ECCO), and the San Antonio Breast Cancer Meeting were also included. The present review considered only those publications that included at least 19 evaluable patients. Phase I data were not included (except in cases where phase II results were not available), and phase III studies have not yet been reported. Because survival data are typically not reported in phase II trials, we did not include these data in this review; however, this review did include time to disease progression whenever reported, as this endpoint is relevant to these studies.

\section{Results}

\section{Single-Agent Gemcitabine}

Nine studies are available that provide an initial assessment of the single-agent activity and tolerability of gemcitabine in advanced breast cancer [13-21] (table 1). Comparability among the studies is limited by different gemcitabine regimens and conditions of pretreatment. The most frequently applied regimens were doses of 1,000 or $1,200 \mathrm{mg} / \mathrm{m}^{2}$ administered over 4 -week cycles.

Gemcitabine monotherapy has produced overall response rates of up to $37 \%$ in the first-line setting [13-15], $26 \%$ in the second-line setting $[15,17]$, and $13 \%$ in the third-line setting [17]. In studies limited to second- or third-line therapy after anthracycline and/or taxane exposure, response rates have reached 29\% [16, 17, 19]. Median time to progression has varied from 2 to 6 months [13-17, 20, 21].

The considerable difference between response rates produced by the first-line studies of Blackstein and Possinger (37 vs. $14 \%$ ) remains unclear [13, 14]. The lower gemcitabine dose intensity in the Possinger study $\left(994 \mathrm{mg} / \mathrm{m}^{2}\right)$ compared to that of the Blackstein study $\left(1,053 \mathrm{mg} / \mathrm{m}^{2}\right)$ can hardly be claimed as a reason for the lower treatment activity, as the remission rate in the Carmichael study, which reported an even lower dose intensity $\left(775 \mathrm{mg} / \mathrm{m}^{2}\right)$, was nearly twice as high $(25 \%)$. Moreover, the ratios of patients with estrogen receptor positivity and prior adjuvant treatment were lower, and therefore more favorable, in the Possinger study. It should be pointed out, however, that the percentage of patients achieving stable disease was comparatively higher in the Possinger study (57 vs. 49\%). Accordingly, the inhibition of tumor progression (stable disease + overall response) did not differ greatly between the studies $(57+14 \%$ for Possinger and $49+37 \%$ for Blackstein).

In one study in which all patients had failed both anthracycline- and taxane-based chemotherapy, there were no responders, although 6 of the 21 evaluable patients achieved stable disease [21]. The heavy pretreat- 
Table 1. Gemcitabine monotherapy

\begin{tabular}{|c|c|c|c|c|c|c|}
\hline $\begin{array}{l}\text { Reference } \\
\text { (first author) }\end{array}$ & $\begin{array}{l}\text { N/Resp } \\
\text { eval }\end{array}$ & Prior chemotherapy & $\begin{array}{l}\text { Study dose } \\
\mathrm{mg} / \mathrm{m}^{2}\end{array}$ & $\begin{array}{l}\text { Median } \\
\text { TTP, months }\end{array}$ & ORR, \% & WHO grade 3 or 4 toxicity ${ }^{\mathrm{a}}, \mathrm{n}$ or $\%$ of patients \\
\hline $\begin{array}{l}\text { Blackstein } \\
{[13]}\end{array}$ & $39 / 35$ & $\begin{array}{l}\text { 1st-line } 39 \mathrm{pts} \\
\text { Adjuvant } 19 \mathrm{pts}\end{array}$ & $\begin{array}{l}1,200 \mathrm{~d} 1,8 \\
15 \mathrm{q} 4 \mathrm{wks}\end{array}$ & 5.1 & $\begin{array}{l}37 \\
\text { (median } \mathrm{RD}=8.8 \mathrm{mos} \text { ) }\end{array}$ & $\begin{array}{l}\text { G3: neutropenia (9), thrombocytopenia ( } 2) \text {, } \\
\text { ALT (2), cutaneous ( } 1 \text { ), nausea/vomiting ( } 4) \text {; } \\
\text { G4: pulmonary (1), infection (1) }\end{array}$ \\
\hline $\begin{array}{l}\text { Possinger } \\
{[14]}\end{array}$ & $42 / 42$ & $\begin{array}{l}\text { 1st-line } 42 \mathrm{pts} \\
\text { Adjuvant } 10 \mathrm{pts}\end{array}$ & $\begin{array}{l}1,000 \mathrm{~d} 1,8 \\
15 \mathrm{q} 4 \mathrm{wks}\end{array}$ & 3.8 & $\begin{array}{l}14 \\
(\text { median } \mathrm{RD}=5.6)\end{array}$ & $\begin{array}{l}\text { G3/4: nausea/vomiting (6), ALP (1), ALT (8), } \\
\text { neutropenia ( } 8 \text { ), thrombocytopenia (2); } \\
\text { G3 only: ALP (1), AST (2) }\end{array}$ \\
\hline $\begin{array}{l}\text { Carmichael } \\
{[15]}\end{array}$ & $44 / 40$ & $\begin{array}{l}\text { 1st-line } 14 \text { pts } \\
\text { 2nd-line } 19 \text { pts } \\
\text { Anthracyclines } 17 \text { pts } \\
\text { Adjuvant } 7 \text { pts }\end{array}$ & $\begin{array}{l}800 \mathrm{~d} 1,8 \\
15 \mathrm{q} 4 \mathrm{wks}\end{array}$ & $2.1^{\mathrm{b}}$ & $\begin{array}{l}25.0 \\
(\text { median RD }=13.5 \mathrm{mos}) \\
\text { 1st-line: } 36(5 / 14) \\
\text { 2nd-line: } 26(5 / 19)\end{array}$ & $\begin{array}{l}\text { G3/4: neutropenia ( } 30 \%) \text {, leukopenia }(9 \%) \text {; } \\
\text { anemia and thrombocytopenia mininal; } \\
\text { AST ( } 9 \%) \text {, nausea/vomiting }(27 \%) \text {; } \\
\text { G4 only: infection ( } 2 \%) \text {; } \\
\text { G3 only: allergic, rash, cardiac function, alopecia, } \\
\text { altered consiousness ( } 2 \% \text { for each) }\end{array}$ \\
\hline $\begin{array}{l}\text { Valerio } \\
{[16]}\end{array}$ & $26 / 22$ & $\begin{array}{l}\text { 2nd- or 3rd-line } 26 \text { pts } \\
\text { Anthracycline, taxane }\end{array}$ & $\begin{array}{l}1,000 \mathrm{~d} 1,8 \\
15 \mathrm{q} 4 \mathrm{wks}\end{array}$ & NA & 23 & $\begin{array}{l}\text { No grade } 3 \text { or } 4 \text { toxicity was reported; } \\
\text { main toxicities were influenza-like syndrome } \\
\text { with fever (10) and fatigue ( } 8)\end{array}$ \\
\hline $\begin{array}{l}\text { Brodowicz } \\
{[17]}\end{array}$ & $25 / 25$ & $\begin{array}{l}\text { 2nd-line } 9 \text { pts } \\
\text { 3rd-line } 16 \text { pts } \\
\text { Anthracyclines } 25 \text { pts } \\
\text { Taxanes } 6 \text { pts }\end{array}$ & $\begin{array}{l}1,250 \mathrm{~d} 1,8 \\
15 \mathrm{q} 4 \mathrm{wks}\end{array}$ & $\begin{array}{l}3.6 \\
\text { 2nd-line: } 5.1 \\
\text { 3rd-line: } 3.5\end{array}$ & $\begin{array}{l}16 \\
\text { 2nd-line: } 22(2 / 9) \\
\text { 3rd-line: } 13(2 / 16)\end{array}$ & $\begin{array}{l}G 3: \text { thrombocytopenia (1) } \\
G 4: \text { thrombocytopenia (4) }\end{array}$ \\
\hline $\begin{array}{l}\text { Gerson } \\
{[18]}\end{array}$ & $19 / 19$ & $\begin{array}{l}\text { 1st-line } 2 \text { pts } \\
\text { 2nd-line } 6 \text { pts } \\
\text { 3rd-line } 6 \text { pts } \\
>\text { 3rd-line } 5 \text { pts }\end{array}$ & $\begin{array}{l}1,250 \mathrm{~d} 1,8 \\
15 \mathrm{q} 4 \mathrm{wks}\end{array}$ & NA & $\begin{array}{l}42 \\
\text { (median } \mathrm{RD}=8.5 \text { mos) }\end{array}$ & $\begin{array}{l}\text { Hematologic side effects ( } 9 \text {; grade not reported), } \\
\text { grade } 2 / 3 \text { thrombocytopenia ( } 5 \text { ); nausea, vomiting, } \\
\text { dysuria, alopecia ( } 2 \text { each; grade not reported) }\end{array}$ \\
\hline $\begin{array}{l}\text { Spielmann } \\
{[19]}\end{array}$ & $47 / 41$ & $\begin{array}{l}\text { 2nd-line } 32 \text { pts } \\
\text { 3rd-line } 15 \text { pts } \\
\text { ( } \geq 1 \text { anthracycline- } \\
\text { or anthracenedione- } \\
\text { based CT) } \\
\text { Adjuvant } 11 \text { pts }\end{array}$ & $\begin{array}{l}1,200 \mathrm{~d} 1,8 \\
15 \mathrm{q} 4 \mathrm{wks}\end{array}$ & NA & $\begin{array}{l}29 \\
\text { (median } \mathrm{RD}=8.1 \mathrm{mos})\end{array}$ & $\begin{array}{l}G 3 \text { : neutropenia (13), thrombocytopenia (3), } \\
\text { nausea/vomiting (4), cutaneous (1); } \\
\text { G4: neutropenia (1) }\end{array}$ \\
\hline $\begin{array}{l}\text { Schmid } \\
{[20]}\end{array}$ & $20 / 20$ & $\begin{array}{l}\text { 1st-line } 4 \text { pts } \\
\text { 2nd-line } 5 \text { pts } \\
\geq 3 \text { rd-line } 11 \text { pts } \\
\text { Adjuvant } 10 \text { pts } \\
\text { Anthracyclines } 15 \text { pts }\end{array}$ & $\begin{array}{l}\text { 250, 6-hour } \\
\text { infusion, d1, } 8 \\
15 \text { q } 4 \text { wks }\end{array}$ & 6.3 & $\begin{array}{l}25 \\
\geq \text { 3rd-line: } 18(2 / 11)\end{array}$ & $\begin{array}{l}\text { G3 only: leukopenia ( } 4 \% \text { of cycles); AST, } \\
\text { ALT ( } 10 \% \text { of cycles for each); GGT ( } 8 \% \text { of cycles), } \\
\text { nausea/vomiting ( } 4 \% \text { of cycles), alopecia } \\
\text { (3\% of cycles) }\end{array}$ \\
\hline $\begin{array}{l}\text { Smorenburg } \\
{[21]}\end{array}$ & $23 / 21$ & $\begin{array}{l}\text { 2nd-line } 3 \text { pts } \\
\geq 3 \text { rd-line } 20 \text { pts } \\
\text { Failed anthracycline- } \\
\text { and taxane-based CT } \\
\text { Adjuvant } 11 \mathrm{pts}\end{array}$ & $\begin{array}{l}1,200 \mathrm{~d} 1,8 \\
15 \mathrm{q} 4 \mathrm{wks}\end{array}$ & 1.9 & 0 & $\begin{array}{l}\text { G3: neutropenia (3), thrombocytopenia (1), } \\
\text { nausea/vomiting ( } 3 \text { ), cutaneous (2), AST ( } 3 \text { ), } \\
\text { ALT (5); } \\
\text { G4: neutropenia (1) }\end{array}$ \\
\hline
\end{tabular}

Resp eval = Response evaluable; TTP $=$ time to progression; ORR = overall response rate; WHO = World Health Organization;

pts = patients; $\mathrm{RD}=$ response duration; $\mathrm{G}=$ grade; $\mathrm{NA}=$ not available.

a Toxicities reported were grade $\geq 3$ hepatic (transaminases only), cutaneous, nausea/vomiting, alopecia, neutropenia, and thrombocytopenia,

and all grade 4 toxicities.

Median TTP based on a personal communication from J. Carmichael.

ment $(57 \%$ of patients pretreated with at least three lines of chemotherapy) and the extensive metastatic disease (visceral disease in $74 \%$ of patients, and at least three organ systems involved in $57 \%$ of patients) may partly account for this outcome. The fact that patients received a low number of median cycles (2, range 1-8) may also have contributed to the lack of efficacy, even though during infusion, the dose intensity was comparatively adequate (mean dose $/$ infusion $=942 \mathrm{mg} / \mathrm{m}^{2}$ ).

While the above-mentioned trials all used a standard infusion time of $30 \mathrm{~min}$, Schmid et al. [20] extended the infusion duration to $6 \mathrm{~h}$ and reduced the gemcitabine dose to $250 \mathrm{mg} / \mathrm{m}^{2}$ and the cycle length to 3 weeks. This trial, performed in variably pretreated patients $(0-2+$ regi- 
mens) produced an overall response rate of $25 \%$ ( $18 \%$ of patients with $2+$ prior regimens) and a median time to progression of 6.3 months.

As a single agent, gemcitabine is moderately active and well tolerated as first-line and salvage therapy of advanced breast cancer. Toxicity generally consists of mild to moderate myelosuppression with minimal clinical consequences, and minimal nonhematologic toxicity, including hepatic and cutaneous toxicity and alopecia. This tolerability is supported by quality-of-life data that appear essentially unchanged by gemcitabine therapy, with a slight improvement in emotional functioning [14].

On the basis of these findings, gemcitabine adequately fits the expectations of a single agent in the treatment of metastatic breast cancer, and offers a valuable alternative to the palliative treatment of this disease.

\section{Gemcitabine plus Anthracyclines}

Anthracycline-based combination chemotherapy has become a standard in the treatment of metastatic breast cancer. Specifically, the FAC regimen, combining 5-fluorouracil, doxorubicin, and cyclophosphamide, may be considered a landmark in terms of efficacy in the first-line setting. In a long-term follow-up of 1,581 patients, this regimen achieved a response rate of $65 \%$, a complete response rate of $17 \%$, and a median progression-free survival of 11.5 months [23].

Doxorubicin's dose-limiting toxicities are myelosuppression, mucositis, and cumulative cardiac toxicity. When gemcitabine is added to doxorubicin, overlapping toxicity is specifically expected with regard to myelosuppression; however, a weekly instead of a monthly application of doxorubicin may reduce toxicity.

First-line chemotherapy with gemcitabine and doxorubicin was studied in a phase II trial conducted by Perez-Manga et al. [24]. Of the 42 patients enrolled, 13 were chemonaive and 29 had received prior adjuvant chemotherapy, which was anthracycline-based in 8 patients. Gemcitabine $\left(800 \mathrm{mg} / \mathrm{m}^{2}\right)$ and doxorubicin $\left(25 \mathrm{mg} / \mathrm{m}^{2}\right)$ were both given on days 1,8 and 15 of a 4 -week cycle. In 6 patients, the gemcitabine dose was escalated from 800 to $1,000 \mathrm{mg} / \mathrm{m}^{2}$. Due to toxicity, only 30 cycles were applied at the higher dose level, and the study was subsequently resumed at the gemcitabine dose of $800 \mathrm{mg} / \mathrm{m}^{2}$. The overall response rate was $55 \%$ (complete response rate of $7 \%$ ), with $31 \%$ of the patients attaining stable disease. The median response duration was 12 months. Apart from notable hematotoxicity, the regimen was well tolerated, as indicated by a low incidence of patient hospitalization, a reduced need for supportive interventions, and the absence of World Health Organization (WHO) grade 3/4 cardiotoxicity. Pharmacokinetic studies indicated that the disposition of both drugs was unchanged when gemcitabine and doxorubicin were administered on the same day. These data clearly indicate that the combination is active, with response rates comparing favorably to those of anthracycline-based combination regimens.

Excellent results were obtained when the gemcitabine/ doxorubicin combination was assessed as neoadjuvant therapy. Gomez et al. [25] administered up to three cycles of gemcitabine $1,200 \mathrm{mg} / \mathrm{m}^{2}$ and doxorubicin $60 \mathrm{mg} / \mathrm{m}^{2}$, every 3 weeks, followed by surgery or radiotherapy. The overall response rate in 39 chemonaive patients was $95 \%$ (complete clinical response rate of $18 \%$ ). Of the 28 patients who underwent mastectomy because their disease was considered resectable, 3 patients had no evidence of disease upon microscopic evaluation of the mastectomy specimens.

Epirubicin is considered less toxic than doxorubicin, which may allow higher doses in combination regimens. The combination of gemcitabine and epirubicin has been investigated in two phase I trials that have been continued in phase II trials. Using a fixed dose of gemcitabine $\left(1,000 \mathrm{mg} / \mathrm{m}^{2}\right)$, one study established the recommended dose of epirubicin at $15 \mathrm{mg} / \mathrm{m}^{2}$, with both drugs given on days 1,8 and 15 of a 4 -week cycle [26]. The other phase I trial evaluated a 3-week schedule of gemcitabine given on days 1 and 8 and epirubicin on day 1 [27]. This schedule appeared to be better tolerated than the 4-week schedule, as the maximum tolerated dose was not reached at gemcitabine $1,500 \mathrm{mg} / \mathrm{m}^{2}$ and epirubicin $75 \mathrm{mg} / \mathrm{m}^{2}$. Thus, a phase II trial was initiated that applied a 3-week regimen of gemcitabine $1,500 \mathrm{mg} / \mathrm{m}^{2}$ and epirubicin $90 \mathrm{mg} / \mathrm{m}^{2}$ [28]. A preliminary report of these data in 20 patients (first-line therapy in $48 \%$ of patients) indicated significant and dose-limiting toxicities that may have contributed to the lower than expected response rate (33\%). Therefore, the gemcitabine regimen was changed to $1,250 \mathrm{mg} / \mathrm{m}^{2}$ on days 1 and 4, while the epirubicin regimen and cycle duration remained the same. Fifteen patients received the modified regimen. Of the 14 evaluable patients, the overall response rate was $60 \%$ (updated to $67 \%$ at final analysis [unpubl. data]). This regimen was better tolerated than the initial regimen, with fewer dose reductions, grade 4 toxicities, discontinuations due to adverse events, and serious adverse events. 


\section{Gemcitabine plus Taxanes}

The taxanes docetaxel and paclitaxel promote the formation of tubulin dimers and stabilize microtubules against depolymerization, thereby causing growth inhibition and cell death. The single-agent activities of the taxanes are comparable [5] or superior [2] to that of doxorubicin, the gold standard in breast cancer treatment. In combination therapies, the taxanes have been tested primarily with anthracyclines.

New taxane combinations not based on anthracyclines, however, are sought primarily because patients are increasingly given anthracyclines during adjuvant treatment. Thus, at relapse, patients may have developed anthracycline resistance, as well as cumulative cardiotoxicity with repeated applications of anthracyclines. Furthermore, because paclitaxel prolongs doxorubicin clearance from plasma, its combination with an anthracycline may augment cardiotoxicity.

The rationale for combining gemcitabine with taxanes is not unequivocally supported by preclinical studies. Some results obtained after concurrent or sequential treatment of cell lines with gemcitabine and paclitaxel indicate less than additive or even antagonistic effects [29], while other in vitro results indicate at least additive cytotoxicity [30]. Pharmacologic studies did not reveal any drug interactions at the level of plasma pharmacokinetics [31, 32]; however, paclitaxel appears to increase the accumulation of gemcitabine's active drug metabolite, gemcitabine triphosphate, in mononuclear cells, suggesting that this drug may enhance the cytotoxic effects of gemcitabine in solid tumors [33].

Apart from the apparent inconsistencies of these data, the combination of gemcitabine and paclitaxel is clearly favored on the basis of the different mechanisms of action of the two agents. Moreover, an indication for this drug combination may be appropriate in anthracycline-pretreated patients, in whom high efficacy is still a treatment goal.

\section{Gemcitabine plus Docetaxel}

The combination of gemcitabine and docetaxel has been explored using different regimens and under different pretreatment conditions (table 2). Single-agent docetaxel has produced overall response rates of $40-68 \%$ [34, 35 ] in previously untreated patients and $30-42 \%$ in patients previously exposed to anthracycline chemotherapy [36]. Although more frequent responses are observed at the higher doses of docetaxel, its use is often limited by toxicity. The dominant dose-limiting toxicity of docetaxel is hematotoxicity (mainly neutropenia), followed by other side effects, such as diarrhea and fluid retention. Thus, depending on dose and schedule, significant hematotoxicity may be encountered when gemcitabine is combined with docetaxel [37, 38].

Two studies assessed gemcitabine plus docetaxel in chemonaive [39] and predominantly chemonaive [38] patients using 2- and 4-week regimens, respectively. Pelegri et al. [39] applied gemcitabine at $2,500 \mathrm{mg} / \mathrm{m}^{2}$ and docetaxel at $65 \mathrm{mg} / \mathrm{m}^{2}$, every 14 days. This regimen produced an overall response rate of $72 \%$ and grade $3 / 4$ neutropenia in approximately half of the patients. Kornek et al. [38] administered lower doses of gemcitabine $(1,500$ $\left.\mathrm{mg} / \mathrm{m}^{2}\right)$ and docetaxel $\left(50 \mathrm{mg} / \mathrm{m}^{2}\right)$ at biweekly intervals; granulocyte colony-stimulating factor (G-CSF) was administered depending on patients' granulocyte nadirs. The overall response rate was $64 \%$ for the 25 chemonaive patients and 59\% for all 34 evaluable patients. Median time to progression was 7 months. Hematotoxicity was generally manageable, presumably because of the use of G-CSF, although septicemia occurred in $9 \%$ of patients.

The combination was also assessed as second- or thirdline therapy after relapse or progression with anthracycline and/or taxane chemotherapy [40-43]. These studies treated patients with 3-week regimens of gemcitabine at 900 or $1,000 \mathrm{mg} / \mathrm{m}^{2}$ and docetaxel from 75 to $100 \mathrm{mg} / \mathrm{m}^{2}$. Overall response rates varied from 36 to $72 \%$, with the highest response rate achieved at the $100-\mathrm{mg} / \mathrm{m}^{2}$ docetaxel dose without G-CSF prophylaxis [43]. Median times to progression, reported in three of the four studies, ranged from 6 to 8 months. Interestingly, the combination produced partial responses in 4 patients who progressed during pretreatment with docetaxel or paclitaxel [41], and partial and complete responses in $72 \%$ of patients who either attained stable disease or progressed during initial docetaxel treatment [43].

The highest response rate achieved so far (79\%) occurred with a 4-week application of gemcitabine $800 \mathrm{mg} /$ $\mathrm{m}^{2}$ on days 1,8 and 15 and docetaxel $100 \mathrm{mg} / \mathrm{m}^{2}$ on day 1 [37]. Most patients were pretreated with anthracyclines in the adjuvant setting. Responses were durable with nearly all responders progression-free for more than 6 months. As growth factor use was avoided in an effort to maintain dose intensity, the hematotoxicity was significant $(97 \%$ of patients with grade $3 / 4$ neutropenia) but manageable.

These studies demonstrate that the combination of gemcitabine and docetaxel may serve as a potent salvage regimen after anthracycline and/or taxane pretreatment. Durable responses of up to $79 \%$ in pretreated patients are achievable. Median times to progression (on the order of 7 months) are consistent across various regimens and 
Table 2. Gemcitabine plus docetaxel

\begin{tabular}{|c|c|c|c|c|c|c|}
\hline $\begin{array}{l}\text { Reference } \\
\text { (first author) }\end{array}$ & $\begin{array}{l}\text { N/Resp } \\
\text { eval }\end{array}$ & Prior chemotherapy & $\begin{array}{l}\text { Study dose } \\
\mathrm{mg} / \mathrm{m}^{2}\end{array}$ & $\begin{array}{l}\text { Median } \\
\text { TTP } \\
\text { months }\end{array}$ & ORR, $\%$ & WHO grade 3 or 4 toxicity ${ }^{\mathrm{a}}, \mathrm{n}$ or $\%$ of patients \\
\hline $\begin{array}{l}\text { Laufman } \\
\text { [37] }\end{array}$ & $39 / 39$ & $\begin{array}{l}\text { 1st-line } 7 \text { pts } \\
\text { Adjuvant } 30 \text { pts } \\
\text { Both } 2 \text { pts } \\
\text { Anthracyclines } 33 \text { pts }\end{array}$ & $\begin{array}{l}\text { Gem } 800 \mathrm{~d} 1,8,15 \\
\text { Doc } 100 \mathrm{~d} 1 \\
\text { q } 4 \text { wks }\end{array}$ & NA & 79 & $\begin{array}{l}\text { G3/4: neutropenia ( } 39 \text { neutropenic fever in } 3 \text { ), } \\
\text { mucositis ( } 2 \text { ); } \\
\text { G3 only: thrombocytopenia (1), } \\
\text { nausea/vomiting (2) }\end{array}$ \\
\hline $\begin{array}{l}\text { Kornek } \\
{[38]}\end{array}$ & $52 / 34$ & $\begin{array}{l}\text { 1st-line } 43 \text { pts } \\
\text { 2nd-line } 9 \text { pts }\end{array}$ & $\begin{array}{l}\text { Gem } 1,500 \mathrm{~d} 1,15 \\
\text { Doc } 50 \mathrm{~d} 1,15 \\
\text { q } 4 \text { wks } \\
\text { G-CSF }\end{array}$ & 7 & $\begin{array}{l}59 \\
\text { (median RD = } 5 \text { mos) } \\
\text { 1st-line: } 64(16 / 25)\end{array}$ & $\begin{array}{l}\text { G3: neutropenia (4); } \\
\text { G4: neutropenia (6); } \\
\text { Mild to moderate (grade } N A) \text { : alopecia }(68 \%) \text {, } \\
\text { nausea/vomiting ( } 35 \%) \text {, skin reactions }(35 \%) \text {, } \\
\text { septicemia }(9 \%)\end{array}$ \\
\hline $\begin{array}{l}\text { Pelegri } \\
{[39]}\end{array}$ & $\begin{array}{l}36 / 25 \\
(29 \text { toxicity } \\
\text { eval) }\end{array}$ & $\begin{array}{l}\text { 1st-line } 36 \mathrm{pts} \\
\text { Adjuvant } 14 \mathrm{pts} \\
\text { Anthracyclines } 10 \mathrm{pts}\end{array}$ & $\begin{array}{l}\text { Gem } 2,500 \mathrm{~d} 1 \\
\text { Doc } 65 \mathrm{~d} 1 \\
\text { q } 2 \text { wks }\end{array}$ & NA & 72 & $\begin{array}{l}\text { G3: neutropenia ( } 7 \text {; febrile } 1) \text {, } \\
\text { thrombocytopenia (1), liver ( } 2 \text { ); } \\
\text { G4: neutropenia ( } 6 \text { ) }\end{array}$ \\
\hline $\begin{array}{l}\text { Brandi } \\
{[40]}\end{array}$ & $37 / 30$ & $\begin{array}{l}\text { 2nd-line } 13 \mathrm{pts} \\
\geq 3 \text { rd-line } 17 \mathrm{pts} \\
\text { (all pts failed on, } \\
\text { or relapsed after, } \\
\text { first-line anthra- } \\
\text { cycline-based CT) }\end{array}$ & $\begin{array}{l}\text { Gem } 1,000 \mathrm{~d} 1,8 \\
\text { Doc } 80 \mathrm{~d} 8 \\
\text { q } 3 \text { wks }\end{array}$ & 6 & $\begin{array}{l}60 \\
(\text { median } \mathrm{RD}=5 \mathrm{mos})\end{array}$ & $\begin{array}{l}G 3 / 4 \text { : neutropenia }(33 \%) \text {, thrombocytopenia }(4 \%) \text {, } \\
\text { nausea/vomiting }(4 \%) \text {; } \\
G 2 / 3 \text { : alopecia }(93 \%)\end{array}$ \\
\hline $\begin{array}{l}\text { Mavroudis } \\
{[41]}\end{array}$ & $52 / 52$ & $\begin{array}{l}\text { 2nd-line } 27 \mathrm{pts} \\
\geq 3 \text { rd-line } 25 \mathrm{pts} \\
\text { (all pts failed on, } \\
\text { or relapsed after, } \\
\text { first-line anthra- } \\
\text { cycline-based CT) } \\
\text { Neoadjuvant } 6 \text { pts } \\
\text { Adjuvant } 23 \text { pts } \\
\text { Taxanes } 25 \text { pts }\end{array}$ & $\begin{array}{l}\text { Gem } 900 \text { d } 1,8 \\
\text { Doc } 100 \mathrm{~d} 8 \\
\text { q } 3 \text { wks } \\
\text { G-CSF (mandatory) }\end{array}$ & 8 & $\begin{array}{l}54 \\
\text { (median } \mathrm{RD}=3.6 \mathrm{mos}) \\
\text { Prior taxanes: } 44 \\
(11 / 25 \mathrm{pts})\end{array}$ & $\begin{array}{l}\text { G3: neutropenia (10; neutropenic fever in } 4) \text {, } \\
\text { thrombocytopenia ( } 9 \text { ), nausea/vomiting (1); } \\
\text { G4: neutropenia (5), thrombocytopenia ( } 2 \text { ) }\end{array}$ \\
\hline $\begin{array}{l}\text { Fountzilas } \\
{[42]}\end{array}$ & $40 / 39$ & $\begin{array}{l}\text { 2nd-line } 20 \text { pts } \\
\text { Adjuvant } 3 \text { pts } \\
\text { Both } 16 \text { pts } \\
\text { (all anthracycline- } \\
\text { resistant) }\end{array}$ & $\begin{array}{l}\text { Gem } 1,000 \mathrm{~d} 1,8 \\
\text { Doc } 75 \mathrm{~d} 1 \\
\text { q } 3 \text { wks } \\
\text { G-CSF }\end{array}$ & 7 & $\begin{array}{l}36 \\
\text { (median RD = } 10.3 \mathrm{mos}) \\
\text { 2nd-line: } 35(7 / 20) \\
\text { Adjuvant: } 33(1 / 3) \\
\text { Both: } 38(6 / 16)\end{array}$ & $\begin{array}{l}\text { G3: neutropenia ( } 7 \text {; febrile neutropenia } 7 \text { ), } \\
\text { thrombocytopenia (1), nausea/vomiting ( } 3 \text { ), } \\
\text { alopecia ( } 30) \text {, dermatitis (1); } \\
\text { G4: neutropenia (12), thrombocytopenia (1) }\end{array}$ \\
\hline $\begin{array}{l}\text { Alexopoulos } \\
{[43]}\end{array}$ & $36 / 36$ & $\begin{array}{l}\text { 2nd- or 3rd-line } \\
\text { (anthracycline- } \\
\text { containing regimens } \\
\text { plus taxanes in } \\
50 \% \text { of pts) }\end{array}$ & $\begin{array}{l}\text { Gem } 900 \text { d } 1,8 \\
\text { Doc } 100 \\
\text { (schedule NA) } \\
\text { q } 3 \text { wks }\end{array}$ & NA & $\begin{array}{l}72 \\
(\text { median } \mathrm{RD}=3.2 \mathrm{mos})\end{array}$ & None reported \\
\hline $\begin{array}{l}\text { Resp eval } \\
\text { Gem = ge } \\
\text { Toxicities } \\
\text { and all gra }\end{array}$ & $\begin{array}{l}=\text { Response } \mathrm{e} \\
\text { mcitabine; D } \\
\text { reported wer }\end{array}$ & $\begin{array}{l}\text { valuable; TTP }=\text { time to } \\
\text { oc }=\text { docetaxel; G-CSF }= \\
\text { e grade } \geq 3 \text { hepatic (tran }\end{array}$ & $\begin{array}{l}\text { orogression; ORR = ov } \\
\text { granulocyte colony-sti } \\
\text { aminases only), cutan }\end{array}$ & $\begin{array}{l}\text { verall resp } \\
\text { imulating } \\
\text { heous, nau }\end{array}$ & $\begin{array}{l}\text { se rate; pts = patients; RD } \\
\text { ctor; CT = chemotherapy; } 1 \\
\text { a/vomiting, alopecia, neutr }\end{array}$ & $\begin{array}{l}\text { response duration; } \mathrm{G}=\text { grade; } \\
=\text { not available. } \\
\text { enia, and thrombocytopenia, }\end{array}$ \\
\hline
\end{tabular}

conditions of pretreatment. These results are an improvement over those of single-agent docetaxel, and could indicate clinical synergism, particularly because patients responded to gemcitabine/docetaxel even after pretreatment, and in some cases after progressing during docetaxel treatment. The toxicity of the combination is manageable, with hematotoxicity appearing dependent on the dose and schedule of the regimen.

\section{Gemcitabine plus Paclitaxel}

Single-agent paclitaxel has produced overall response rates of $32-62 \%$ in previously untreated patients [44-46], and $6-48 \%$ in anthracycline-resistant patients [47-49]. The addition of gemcitabine to paclitaxel is especially interesting because paclitaxel is less hematotoxic than docetaxel at standard doses for each. Overlapping hematotoxicity between gemcitabine and paclitaxel should therefore be less pronounced. The feasibility of combin- 
Table 3. Gemcitabine plus paclitaxel

\begin{tabular}{|c|c|c|c|c|c|c|}
\hline $\begin{array}{l}\text { Reference } \\
\text { (first author) }\end{array}$ & $\begin{array}{l}\text { N/Resp } \\
\text { eval }\end{array}$ & Prior chemotherapy & $\begin{array}{l}\text { Study dose } \\
\mathrm{mg} / \mathrm{m}^{2}\end{array}$ & $\begin{array}{l}\text { Median } \\
\text { TTP } \\
\text { months }\end{array}$ & ORR, \% & WHO grade 3 or 4 toxicity ${ }^{\mathrm{a}}, \mathrm{n}$ or $\%$ of patients \\
\hline $\begin{array}{l}\text { Colomer } \\
{[50]}\end{array}$ & $\begin{array}{l}43 / 38 \\
\text { (34 toxicity } \\
\text { eval) }\end{array}$ & $\begin{array}{l}\text { 1st-line } 43 \text { pts } \\
\text { Adjuvant } 24 \text { pts }\end{array}$ & $\begin{array}{l}\text { Gem } 2,500 \mathrm{~d} 1 \\
\text { Pac } 150(3 \mathrm{hr}) \mathrm{d} 1 \\
\text { q } 2 \text { wks }\end{array}$ & NA & 68 & $\begin{array}{l}\text { G3: neutropenia (5), thrombocytopenia (2), } \\
\text { nausea (2), vomiting ( } 2 \mathrm{pts} \text { ), } \\
\text { liver transaminases (2); } \\
\text { G4: neutropenia (6), lymphocytes (2), } \\
\text { leukopenia (3), fever and neutropenia (1) }\end{array}$ \\
\hline $\begin{array}{l}\text { Delfino } \\
{[51]}\end{array}$ & $42 / 42$ & $\begin{array}{l}\text { 1st-line } 42 \text { pts } \\
\text { Adjuvant } 27 \mathrm{pts}\end{array}$ & $\begin{array}{l}\text { Gem } 1,200 \mathrm{~d} 1,8 \\
\text { Pac } 175(3 \mathrm{~h}) \mathrm{d} 1 \\
\text { q } 3 \text { wks }\end{array}$ & NA & $\begin{array}{l}55 \\
\text { (median } \mathrm{RD}=19 \mathrm{mos})\end{array}$ & $\begin{array}{l}\text { Leukopenia and thrombocytopenia ( } 6 \text { each), } \\
\text { mucositis ( } 7 \text { ) }\end{array}$ \\
\hline $\begin{array}{l}\text { Genot } \\
{[52]}\end{array}$ & $40 / 36$ & 1st-line 40 pts & $\begin{array}{l}\text { Gem } 1,200 \mathrm{~d} 1,8 \\
\text { Pac } 175(3 \mathrm{~h}) \mathrm{d} 1 \\
\text { q } 3 \text { wks }\end{array}$ & 7.5 & $\begin{array}{l}42 \\
(\text { median } \mathrm{RD}=11.5 \mathrm{mos})\end{array}$ & $\begin{array}{l}G 4: \text { neutropenia ( } 41 \text { events); } \\
G 3: \text { leukopenia ( } 52 \text { events); neutropenia } \\
\text { ( } 24 \text { events) }\end{array}$ \\
\hline $\begin{array}{l}\text { Sanchez } \\
{[53]}\end{array}$ & $44 / 44$ & $\begin{array}{l}\text { 2nd-line } 44 \text { pts } \\
\text { Anthracyclines } 41 \text { pts } \\
\text { Pac } 9 \text { pts }\end{array}$ & $\begin{array}{l}\text { Gem } 2,500 \mathrm{~d} 1,15 \\
\text { Pac } 135(3 \mathrm{~h}) \mathrm{d} 1,15 \\
\text { q } 4 \text { wks }\end{array}$ & 7 & 45 & G3/4: hematologic toxicity ( $15 \%$ of cycles) \\
\hline $\begin{array}{l}\text { Murad } \\
{[54]}\end{array}$ & $29 / 29$ & $\begin{array}{l}\text { Second or third } \\
\text { relapse during } \\
\text { anthracycline- } \\
\text { based CT }\end{array}$ & $\begin{array}{l}\text { Gem } 1,000 \mathrm{~d} 1,8 \\
\text { Pac } 175(3 \mathrm{~h}) \mathrm{d} 1 \\
\text { q } 3 \text { wks } \\
\text { (initial regimen was Gem } \\
1,000 \text { on d1, } 8,15 \text { with } \\
\text { same Pac dose over } \\
4 \text { weeks. This was changed } \\
\text { to } 3 \text { weeks with the day-15 } \\
\text { dose dropped after unac- } \\
\text { ceptable toxicity (thrombo- } \\
\text { cytopenia) in the first } 5 \text { pts) }\end{array}$ & NA & $\begin{array}{l}55 \\
\text { (median } \mathrm{RD}=8 \mathrm{mos})\end{array}$ & $\begin{array}{l}G 3: \text { neutropenia ( } 6 \% \text { of cycles), thrombo- } \\
\text { cytopenia ( } 5 \% \text { of cycles), nausea/vomiting } \\
\text { ( } 6 \% \text { of cycles), alopecia ( } 76 \% \text { of cycles); } \\
G 4: \text { neutropenia ( } 3 \% \text { of cycles; } 2 \text { pts w/fever), } \\
\text { thrombocytopenia ( } 3 \% \text { of cycles), infection } \\
\text { ( } 3 \% \text { of cycles) }\end{array}$ \\
\hline
\end{tabular}

Resp eval = Response evaluable; TTP = time to progression; ORR = overall response rate; WHO = World Health Organization; pts = patients;

$\mathrm{RD}=$ response duration; $\mathrm{G}=$ grade $; \mathrm{Gem}=$ gemcitabine $; \mathrm{Pac}=$ paclitaxel; NA = not available

a Toxicities reported were grade $\geq 3$ hepatic (transaminases only), cutaneous, nausea/vomiting, alopecia, neutropenia, and thrombocytopenia, and all grade 4 toxicities.

ing gemcitabine with paclitaxel has been assessed in chemonaive and pretreated patients (table 3 ).

Colomer et al. [50] administered a first-line regimen of gemcitabine $2,500 \mathrm{mg} / \mathrm{m}^{2}$ and paclitaxel $150 \mathrm{mg} / \mathrm{m}^{2}$ both given on day 1 , every 2 weeks. Most patients completed adjuvant chemotherapy $\leq 12$ months before relapse. The overall response rate in 38 evaluable patients was $68 \%$. Hematologic toxicity was mainly limited to neutropenia (32\% of patients), with only one report of neutropenic fever.

Two additional studies of the combination as first-line therapy for metastatic disease $[51,52]$ yielded response rates of 55 and $42 \%$, respectively, using identical 3-week regimens of $1,200 \mathrm{mg} / \mathrm{m}^{2}$ gemcitabine and $175 \mathrm{mg} / \mathrm{m}^{2}$ paclitaxel. Responses were durable with median response durations of about 1 year or better (19 and 11.5 months, respectively). One study reported a median time to progression of 7.5 months [52].

Gemcitabine in Advanced and Metastatic

Breast Cancer
Sanchez et al. [53] assessed a 4-week schedule of paclitaxel at $135 \mathrm{mg} / \mathrm{m}^{2}$ and gemcitabine at $2,500 \mathrm{mg} / \mathrm{m}^{2}$, with both drugs delivered on days 1 and 15. Patients were heavily pretreated for metastatic disease $(93 \%$ with anthracycline-based regimens). An overall response rate of $45 \%$ was reached, but about a third of the patients required growth factors to continue receiving the planned dosing schedule. The median time to progression was 7 months.

Murad et al. [54] conducted a trial in patients who relapsed a second or third time after anthracycline-based chemotherapy. Initially, a 4-week regimen of gemcitabine at $1,000 \mathrm{mg} / \mathrm{m}^{2}$ and paclitaxel at $175 \mathrm{mg} / \mathrm{m}^{2}$ was given on day 1 (paclitaxel only), 8 and 15. Severe thrombocytopenia was observed in the first 5 patients, necessitating a change in the treatment schedule from 4 to 3 weeks, with the day- 15 gemcitabine dose withdrawn. In these heavily pretreated patients, the overall response rate was $55 \%$

Oncology 2003;64:191-206 
Table 4. Gemcitabine plus vinorelbine

\begin{tabular}{|c|c|c|c|c|c|c|}
\hline $\begin{array}{l}\text { Reference } \\
\text { (first author) }\end{array}$ & $\begin{array}{l}\text { N/Resp } \\
\text { eval }\end{array}$ & Prior chemotherapy & $\begin{array}{l}\text { Study dose } \\
\mathrm{mg} / \mathrm{m}^{2}\end{array}$ & $\begin{array}{l}\text { Median } \\
\text { TTP, months }\end{array}$ & ORR, $\%$ & WHO grade 3 or 4 toxicity ${ }^{\mathrm{a}}$, $\mathrm{n}$ or $\%$ of patients \\
\hline $\begin{array}{l}\text { Haider } \\
{[58]}\end{array}$ & $45 / 45$ & $\begin{array}{l}\text { 1st-line } 45 \text { pts } \\
\text { 2nd-line } 15 \text { pts } \\
\text { (anthracyclines } 10 \mathrm{pts} \text { ) }\end{array}$ & $\begin{array}{l}\text { Gem } 1,000 \mathrm{~d} 1, \\
15,21 \\
\text { Vin } 40 \mathrm{~d} 1,21 \\
\text { q } 4 \text { wks } \\
\text { G-CSF }\end{array}$ & $\begin{array}{l}8.5 \\
\text { 1st-line: } 9.5 \\
\text { 2nd-line: } 7\end{array}$ & $\begin{array}{l}52 \\
\text { (median RD = } 8.5 \text { mos) } \\
\text { 1st-line } 56(25 / 45) \\
\text { 2nd-line } 40(6 / 15)\end{array}$ & $\begin{array}{l}\text { G3: neutropenia (9), nausea/vomiting (3); } \\
\text { G4: neutropenia (2), leukopenia (1) }\end{array}$ \\
\hline $\begin{array}{l}\text { Valenza } \\
{[59]}\end{array}$ & $29 / 29$ & $\begin{array}{l}\text { 2nd-line } 29 \text { pts } \\
\text { Anthracycline/taxane } \\
\text { Adjuvant } 25 \text { pts }\end{array}$ & $\begin{array}{l}\text { Gem } 1,000 \mathrm{~d} 1, \\
8,15 \\
\text { Vin } 25 \mathrm{~d} 1,8 \\
\text { q } 4 \text { wks }\end{array}$ & $\begin{array}{l}6.8+ \\
\text { (mean) }\end{array}$ & 48 & G3: thrombocytopenia (3) \\
\hline $\begin{array}{l}\text { Stathopoulos } \\
{[60]}\end{array}$ & $51 / 50$ & $\begin{array}{l}\text { 2nd-line } 51 \text { pts } \\
\text { Anthracycline-based CT } \\
\text { Anthracycline/taxane } 25 \text { pts }\end{array}$ & $\begin{array}{l}\text { Gem } 1,000 \mathrm{~d} 115 \\
\text { Vin } 25 \mathrm{~d} 1,15 \\
\text { q } 4 \text { wks }\end{array}$ & 6 & $\begin{array}{l}54 \\
(\text { median } \mathrm{RD}=6 \mathrm{mos})\end{array}$ & G3/4: neutropenia (4) \\
\hline $\begin{array}{l}\text { Nicolaides } \\
{[61]}\end{array}$ & $31 / 27$ & $\begin{array}{l}\text { 2nd-line } 31 \mathrm{pts} \\
\text { Taxane-based CT }\end{array}$ & $\begin{array}{l}\text { Gem } 1,000 \mathrm{~d} 1,8 \\
\text { Vin } 30 \mathrm{~d} 1,8 \\
\text { q } 3 \text { wks }\end{array}$ & 3.5 & $\begin{array}{l}22 \\
(\text { median } \mathrm{RD}=6 \mathrm{mos})\end{array}$ & $\begin{array}{l}\text { G3/4: neutropenia (15), thrombocytopenia (1), } \\
\text { paralytic ileus (1), rash (3) }\end{array}$ \\
\hline $\begin{array}{l}\text { Mariani } \\
{[62]^{\mathrm{b}}}\end{array}$ & $31 / 27$ & $\begin{array}{l}\text { 2nd- or } \geq 3 \text { rd-line } \\
\text { (at least } 90 \% \text { of pts) } \\
\text { Anthracycline- and/or } \\
\text { taxane-based CT }\end{array}$ & $\begin{array}{l}\text { Gem } 1,200 \mathrm{~d} 1,8 \\
\text { Vin } 30 \mathrm{~d} 1,8 \\
\text { q } 3 \text { wks }\end{array}$ & NA & 22 & G3/4: neutropenia (15), thrombocytopenia (2) \\
\hline $\begin{array}{l}\text { Donadio } \\
{[63]}\end{array}$ & $26 / 23$ & $\begin{array}{l}\text { 2nd-line } 21 \mathrm{pts} \\
\text { 3rd-line } 5 \mathrm{pts} \\
\text { Anthracyclines } 12 \mathrm{pts} \\
\text { Adjuvant and/or } \\
\text { neoadjuvant } 19 \mathrm{pts}\end{array}$ & $\begin{array}{l}\text { Gem } 1,000 \mathrm{~d} 1,8 \\
\text { Vin } 25 \mathrm{~d} 1,8 \\
\text { q } 3 \text { wks }\end{array}$ & NA & 39 & None reported \\
\hline $\begin{array}{l}\text { Moser } \\
{[64]}\end{array}$ & $\begin{array}{l}69 / 30 \\
(38 \text { tox- } \\
\text { icity } \\
\text { eval) }\end{array}$ & $\begin{array}{l}\text { 1st-line } 25 \mathrm{pts} \\
\text { 2nd-line } 13 \mathrm{pts} \\
\text { Anthracycline- and/or } \\
\text { taxane-based CT } 11 \mathrm{pts}\end{array}$ & $\begin{array}{l}\text { Gem } 1,200 \mathrm{~d} 1,8 \\
\text { Vin } 25 \mathrm{~d} 1,8 \\
\text { q } 3 \text { wks }\end{array}$ & NA & 30 & G3/4: neutropenia (7), phlebitis (1) \\
\hline $\begin{array}{l}\text { Gokmen } \\
{[65]}\end{array}$ & $26 / 22$ & $\begin{array}{l}\text { 1st-line } 6 \text { pts } \\
\text { 2nd-line } 13 \text { pts } \\
\text { 3rd-line } 7 \text { pts } \\
\text { Anthracycline refractory or } \\
\text { resistant } 96 \% \text { of pts }\end{array}$ & $\begin{array}{l}\text { Gem } 1,200 \mathrm{~d} 1,8 \\
\text { Vin } 30 \mathrm{~d} 1,8 \\
\text { q } 3 \text { wks }\end{array}$ & 5.5 & 45 & $\begin{array}{l}\text { G3: thrombocytopenia (4), phlebitis (2); } \\
\text { G4: leukopenia (1) }\end{array}$ \\
\hline
\end{tabular}

Resp eval = Response evaluable; TTP = time to progression; ORR = overall response rate; WHO = World Health Organization; $\mathrm{CT}=$ chemotherapy;

$\mathrm{Gem}=$ gemcitabine; Vin = vinorelbine; G-CSF = granulocyte colony-stimulating factor; $\mathrm{RD}=$ response duration; $\mathrm{G}=$ grade; pts = patients; NA = not available.

a Toxicities reported were grade $\geq 3$ hepatic (transaminases only), cutaneous, nausea/vomiting, alopecia, neutropenia, and thrombocytopenia,

and all grade 4 toxicities.

b For this phase I/II study, only phase II data are presented.

with a median duration of response of 8 months. Grade $3 / 4$ neutropenia was reported in $30 \%$ of patients, which was accompanied by neutropenic fever in $14 \%$ of patients.

These results demonstrate that the combination of gemcitabine and paclitaxel is effective in both chemonaive and heavily pretreated patients. High and durable responses across different regimens are achievable. Toxicities are manageable, with neutropenia being the main toxicity. The response rate of $55 \%$ obtained in anthracycline-pretreated patients [54] may be particularly appre- ciated in view of the low response rate to single-agent paclitaxel (16\%) observed after doxorubicin failure in a phase III study [3]. Although preclinical data do not provide an established basis for synergistic drug interaction, these clinical data clearly support the use of this combination.

\section{Gemcitabine plus Vinorelbine}

Vinorelbine exerts antitumor activity through destabilization of microtubules. While this drug has shown good efficacy as first-line treatment (40-60\%) [55], activity 
after anthracycline pretreatment has only been moderate $(16 \%)[56,57]$. Vinorelbine is characterized by a favorable profile of side effects, with low rates of nausea and emesis, and almost no alopecia. The dose-limiting toxicity of vinorelbine is in the form of noncumulative hematotoxicity. The potential of adding gemcitabine to vinorelbine, two well-tolerated agents with different mechanisms of action, has been explored in various phase II studies (table 4).

Haider et al. [58] conducted the only study that evaluated gemcitabine plus vinorelbine separately as first-line therapy. In a subgroup of 45 chemonaive patients, a 4week regimen of gemcitabine $1,000 \mathrm{mg} / \mathrm{m}^{2}$ and vinorelbine $40 \mathrm{mg} / \mathrm{m}^{2}$ together with G-CSF support produced an overall response rate of $56 \%$ and a median time to progression of 9.5 months. In the remaining 15 patients who were pretreated with palliative therapy (including anthracyclines in 10 patients), lower values of response rate (40\%) and median time to progression (7 months) were reported.

Two additional studies that applied 4-week regimens of gemcitabine plus vinorelbine, but solely as second-line therapy (after anthracyclines \pm taxanes), achieved response rates of 48 and 54\%, respectively [59, 60]. Threeweek regimens using identical schedules but slightly different doses of gemcitabine or vinorelbine as second- or third-line therapy produced lower response rates of $22 \%$ $[61,62]$ or $39 \%$ [63].

Two studies evaluated 3-week regimens in variably pretreated patients ( $0-2$ prior regimens) $[64,65]$. Preliminary data reported by Moser et al. [64] in 38 eligible patients (66\% chemonaive), indicated an overall remission rate of $30 \%$ in 30 evaluable patients. Gokmen et al. [65] used an identical regimen except for a slightly higher dose of vinorelbine ( 30 vs. $25 \mathrm{mg} / \mathrm{m}^{2}$ ). Most patients had undergone one to two prior chemotherapies, although the pretreatment pattern was not uniform with the combination administered as first-line therapy in 23\%, as secondline therapy in 50\%, and as third-line therapy in $27 \%$ of patients. Anthracycline resistance was described in $31 \%$ of patients, and anthracycline-refractory disease in $65 \%$ of patients. In these predominantly anthracycline-exposed patients, the combination of gemcitabine and vinorelbine achieved a response rate of $45 \%$ accompanied by a median time to progression of 5.5 months.

These studies demonstrate that the combination of gemcitabine and vinorelbine is active not only as first-line treatment but also after pretreatment with anthracyclineor anthracycline/taxane-based regimens. Response rates of up to $54 \%$ in anthracycline-pretreated patients are achievable. Hematotoxicity, the main toxicity of this combination, appears manageable and generally does not require the use of hematopoietic growth factors.

\section{Gemcitabine plus Vindesine}

Vindesine is a known spindle toxin, with a mechanism of action comparable to vinorelbine. While drug application every 3 weeks may be considered an advantage of this agent, neurotoxicity is more pronounced compared to that of vinorelbine. Cazzaniga et al. [66] conducted a multicenter phase II trial evaluating a 3-week regimen of gemcitabine $\left(1,000 \mathrm{mg} / \mathrm{m}^{2}\right.$ on days 1 and 8$)$ combined with vindesine $\left(3 \mathrm{mg} / \mathrm{m}^{2}\right.$ on day 1$)$. All but 1 of the 42 enrolled patients had prior first-line therapy (21 had anthracyclines with or without taxanes and three had taxanes only). The overall response rate in 25 evaluable patients was $32 \%$ (all partial responses); $24 \%$ of patients reached stable disease. Grade 3/4 neutropenia was observed in $44 \%$ of the patients.

\section{Gemcitabine plus Cisplatin}

Until recently, cisplatin did not play a significant role in breast cancer treatment. In five studies analyzing 119 pretreated patients, a mean response rate of $7 \%$ was observed [67-71]. Despite its low activity as a single agent in salvage therapy, cisplatin appears to be a viable partner for combination treatment. In fact, it has been successfully tested together with docetaxel, vinorelbine, anti-HER2/ neu antibody, and recently with gemcitabine (table 5).

Three lines of argument support this combination. First, breast cancer patients treated with standard regimens in the adjuvant or palliative setting will not have been exposed to gemcitabine or cisplatin in most cases. Consequently, the probability of pretreatment-induced drug resistance to these drugs is low. Second, synergistic cytotoxicity has been observed in vitro when adequate repair of cisplatin-induced DNA damage was prevented by gemcitabine [72]. Third, synergistic interaction between anti-HER2/neu antibody and cisplatin has been reported both experimentally and in breast cancer patients [73]. Therefore, the addition of trastuzumab, an anti-HER2/neu antibody, to gemcitabine/cisplatin might form an effective triplet combination, and thus may offer another treatment option after anthracycline and taxane pretreatment.

Nagourney et al. [74] first described the in vitro synergism of gemcitabine and cisplatin in patients with breast cancer. Synergistic cytotoxicity was observed in $73 \%$ of 225 tumor probes $(68 \%$ originating from pretreated patients). As first-line therapy, the combination of gemcita- 
Table 5. Gemcitabine plus cisplatin

\begin{tabular}{|c|c|c|c|c|c|c|}
\hline $\begin{array}{l}\text { Reference } \\
\text { (first author) }\end{array}$ & $\begin{array}{l}\text { N/Resp } \\
\text { eval }\end{array}$ & Prior chemotherapy & $\begin{array}{l}\text { Study dose } \\
\mathrm{mg} / \mathrm{m}^{2}\end{array}$ & $\begin{array}{l}\text { Median } \\
\text { TTP, months }\end{array}$ & ORR, $\%$ & WHO grade 3 or 4 toxicity, $\mathrm{n}$ or $\%$ of patients \\
\hline $\begin{array}{l}\text { Calderillo } \\
\text { Ruiz } \\
{[75]}\end{array}$ & $31 / 31$ & 1st-line $31 \mathrm{pts}$ & $\begin{array}{l}\text { Gem } 1,200 \mathrm{~d} 1,8 \\
\text { Cis } 75 \mathrm{~d} 1 \\
\text { q } 3 \text { wks }\end{array}$ & NA & 80 & $\begin{array}{l}G 3 / 4 \text { : neutropenia ( } 20 \% \text { of cycles), anemia } \\
\text { ( } 3 \% \text { of cycles), thrombocytopenia ( } 2 \% \text { of cycles), } \\
\text { nausea/vomiting ( } 17 \% \text { of cycles) }\end{array}$ \\
\hline $\begin{array}{l}\text { Nagourney } \\
{[76]}\end{array}$ & $30 / 30$ & $\begin{array}{l}\text { 2nd- to } 6 \text { th-line } \\
\text { Anthracyclines } 14 \text { pts } \\
\text { Taxanes } 20 \text { pts }\end{array}$ & $\begin{array}{l}\text { Gem } 1,000 \text { Cis } 30 \\
\mathrm{~d} 1,8,15 \mathrm{q} 4 \text { wks } \\
\text { (after } 12 \text { pts, } \\
\text { regimen changed } \\
\text { to Gem } 750, \text { Cis } 30 \\
\text { d } 1,8 \text { q } 3 \text { wks) }\end{array}$ & $\begin{array}{l}3.5 \\
\text { 2nd- or 3rd-line: } 5.5 \\
>4 \text { th-line: } 3.5\end{array}$ & 50 & $\begin{array}{l}G 3: \text { leukopenia ( } 17 \% \text { of cycles), neutropenia } \\
\text { ( } 11 \% \text { of cycles), anemia ( } 6 \% \text { of cycles), } \\
\text { thrombocytopenia ( } 33 \% \text { of cycles); } \\
G 4: \text { leukopenia ( } 2 \% \text { of cycles), neutropenia } \\
\text { ( } 4 \% \text { of cycles), thrombocytopenia ( } 14 \% \text { of cycles) }\end{array}$ \\
\hline $\begin{array}{l}\text { Chaudry } \\
{[77]}\end{array}$ & $28 / 28$ & $\begin{array}{l}\text { 2nd-line } 28 \text { pts } \\
\text { Anthracycline- and } \\
\text { taxane-based CT }\end{array}$ & $\begin{array}{l}\text { Gem } 1,000 \mathrm{~d} 1,8,15 \\
\text { Cis } 25 \mathrm{~d} 1,8,15 \\
\text { q } 4 \text { wks }\end{array}$ & NA & $\begin{array}{l}39 \\
\text { median RD } \\
=5.3 \mathrm{mos}\end{array}$ & $\begin{array}{l}G 4: \text { thrombocytopenia ( } 12 \% \text { of cycles), } \\
\text { neutropenia ( } 9 \% \text { of cycles), nausea/vomiting } \\
\text { ( } 4.5 \% \text { of cycles) }\end{array}$ \\
\hline $\begin{array}{l}\text { Burch } \\
{[78]}\end{array}$ & $21 / 21$ & $\begin{array}{l}\text { 2nd-line } 21 \text { pts } \\
\text { 3rd-line } 17 \text { pts } \\
\text { Anthracycline or taxane }\end{array}$ & $\begin{array}{l}\text { Gem } 1,000 \mathrm{~d} 1,8,15 \\
\text { Cis } 25 \mathrm{~d} 1,8,15 \\
\text { q } 4 \text { wks }\end{array}$ & 7.1 & 29 & $\begin{array}{l}G 3 \text { : neutropenia ( } 38 \%) \text {, thrombocytopenia }(24 \%) \text {; } \\
G 4: \text { neutropenia }(43 \%) \text {, thrombocytopenia }(38 \%)\end{array}$ \\
\hline $\begin{array}{l}\text { Doroshow } \\
{[79]}\end{array}$ & $55 / 44$ & $\begin{array}{l}\text { M: } 24 \text { pts ( } 21 \text { eval) } \\
\text { 1st-line, } 10 \text { pts; } \\
\text { 2nd-line } 14 \text { pts } \\
\text { H: } 31 \text { pts ( } 23 \text { eval) } \\
\text { > 3rd-line (including } \\
\text { doxorubicin or a taxane) }\end{array}$ & $\begin{array}{l}\text { Gem } 1,000 \mathrm{~d} 2,8 \\
\text { Cis } 25 \mathrm{~d} 1-4 \\
\text { q } 3 \text { wks } \\
\text { G-CSF } \\
\text { (mandatory for } \mathrm{H} \text { ) }\end{array}$ & $\begin{array}{l}\text { 1st-line } 8.3 \mathrm{mos} \\
\text { 2nd-line } 3.7 \mathrm{mos} \\
>\text { 3rd-line } 3.5 \mathrm{mos}\end{array}$ & $\begin{array}{l}34 \\
\text { M } 43 \\
\text { H } 26\end{array}$ & $\begin{array}{l}G 3 \text { : neutropenia (9), thrombocytopenia (19), } \\
\text { vomiting ( } 8 \text { ); } \\
G 4 \text { : neutropenia (30), thrombocytopenia (19), } \\
\text { anemia (5) }\end{array}$ \\
\hline $\begin{array}{l}\text { Galvez } \\
{[80]}\end{array}$ & $41 / 41$ & $\begin{array}{l}\text { 2nd-line } 41 \text { pts } \\
\text { Anthracycline-based CT }\end{array}$ & $\begin{array}{l}\text { Gem } 1,200 \mathrm{~d} 1,8,15 \\
\text { Cis } 50 \mathrm{~d} 1 \\
\text { q } 4 \text { wks }\end{array}$ & 5.2 & $\begin{array}{l}49 \\
\text { median RD } \\
=10.6 \operatorname{mos}\end{array}$ & $\begin{array}{l}\text { G3/4: thrombocytopenia }(47 \%) \text {, neutropenia } \\
(48 \%) \text {, anemia }(42 \%) \text {, nausea/vomiting }(17 \%) \text {, } \\
\text { alopecia }(77 \%) \text {, nephrotoxicity }(9 \%) \text {, neuropathy } \\
(11 \%)\end{array}$ \\
\hline
\end{tabular}

Resp eval $=$ Response evaluable; TTP $=$ time to progression; ORR = overall response rate; WHO = World Health Organization;

$\mathrm{CT}=$ chemotherapy $\mathrm{RD}=$ response duration; $\mathrm{G}=$ grade; $\mathrm{pts}=$ patients; $\mathrm{Gem}=$ gemcitabine Cis = cisplatin; NA = not available;

$\mathrm{M}=$ moderately pretreated; $\mathrm{H}=$ heavily pretreated.

a Toxicities reported were grade $\geq 3$ hepatic (transaminases only), cutaneous, nausea/vomiting, alopecia, neutropenia, and thrombocytopenia, and all grade 4 toxicities.

bine $\left(1,200 \mathrm{mg} / \mathrm{m}^{2}\right)$ and cisplatin $\left(75 \mathrm{mg} / \mathrm{m}^{2}\right)$ given every 3 weeks with cisplatin given once during the cycle (day 1 ) has proven to be highly effective, reaching a response rate of $80 \%$ in one phase II study [75]. Five additional studies performed in moderate to intensively pretreated patients, which used lower doses of cisplatin given repeatedly [7679] or once [80] during 3- or 4-week cycles, demonstrated a median overall response rate of 39\% (range 29-50\%). The toxicity profiles of these regimens were moderate, with thrombocytopenia and neutropenia being the main side effects.

At present, an optimal regimen for the treatment of breast cancer has not been determined in a comparative fashion. To optimize synergy, it was suggested to administer 'repeating doublets' of drugs [81] such as the application used in the Nagourney regimen [76], in which both drugs were given on days 1 and 8. The efficacy as well as the hematotoxicity of this drug application appear favor- able. If outpatient use is preferred, the lower weekly cisplatin dose $\left(30 \mathrm{mg} / \mathrm{m}^{2}\right)$ that can be applied in these regimens offers an additional advantage.

\section{Gemcitabine plus Epirubicin/Paclitaxel (GET) or Doxorubicin/Paclitaxel (GAT)}

Anthracyclines and the taxanes belong to the most active groups of agents used in breast cancer treatment. In principle, there is no evidence that the addition of a third agent to a doublet truly adds to efficacy more than it adds to toxicity. A major goal of the combined application of GET or GAT was to improve the complete response rate, since it was claimed that patients achieving a complete response to first-line treatment may be good candidates for long-term survival [23]. To date, the GET and GAT trials have been performed exclusively in the first-line setting. 
Two phase II studies evaluating GET have been reported $[82,83]$. Both studies used identical 3-week regimens of gemcitabine at $1,000 \mathrm{mg} / \mathrm{m}^{2}$ on days 1 and 4 , epirubicin at $90 \mathrm{mg} / \mathrm{m}^{2}$ on day 1 , and paclitaxel $175 \mathrm{mg} / \mathrm{m}^{2}$ (3-hour infusion) on day 1. A single-institution study by Conte et al. [82] showed that this regimen was highly active, demonstrating an overall response rate of $92 \%$ and a complete response rate of $31 \%$ after six courses. Additionally, 25 of the 36 enrolled patients received high-dose chemotherapy, providing a final overall response rate of $97 \%$ and a complete response rate of $47 \%$. Median time to progression was 21 months (median follow-up 25 months). On the basis of these very optimistic data, GET was subsequently tested in a multicenter setting of 39 patients [83]. After a median of two courses, the overall response rate was $58 \%$, with a complete response rate of $10 \%$. Final response data, after a median of six courses, are not available. The favorable response rates in these two studies were accompanied by considerable hematotoxicity, with WHO grade 3 and 4 neutropenia reported in 64 and $59 \%$ of the patients, respectively.

Sanchez-Rovira et al. [84] observed an overall response rate of $83 \%$ (complete response rate of $44 \%$ ) in a single-institution study of the GAT combination administered to 34 patients over a 4-week schedule (day-2 gemcitabine $2,500 \mathrm{mg} / \mathrm{m}^{2}$ and paclitaxel $135 \mathrm{mg} / \mathrm{m}^{2}$, day- 1 doxorubicin $30 \mathrm{mg} / \mathrm{m}^{2}$ ). The median duration of response and the median time to progression were each 14 months. As with GET, this regimen appears highly active but at the expense of toxicity, with $21 \%$ of cycles reduced or delayed primarily due to neutropenia, despite the use of G-CSF in $66 \%$ of patients.

As a follow-up to this study, Sanchez-Rovira et al. [85, 86] are also conducting a phase II study of the GAT regimen as neoadjuvant therapy in patients with stage II/III invasive breast cancer. Patients received six cycles of GAT prior to surgery and three additional cycles after surgery. An overall response rate of $98 \%$ (complete response rate of $42 \%$ ) has been achieved in 33 assessable patients. The median duration of response was $13+$ months.

\section{Gemcitabine plus 5-Fluorouracil/Leucovorin}

The combination of the two antimetabolites, gemcitabine and 5-fluorouracil, has shown synergistic cytotoxicity in colorectal tumor cell lines in vitro [87]. Meanwhile, much knowledge regarding this combination in pancreatic cancer has been accumulated. While toxicity has been generally moderate, it is unclear whether this drug combination is superior to that of single-agent gemcitabine.
The approach of combining gemcitabine and 5-fluorouracil in breast cancer is primarily based on the good tolerability and efficacy of both drugs in this tumor entity. Thus far, one study of the gemcitabine/5-fluorouracil/leucovorin combination has been conducted. Mulkerin et al. [88] administered gemcitabine $1,000 \mathrm{mg} / \mathrm{m}^{2}, 5$-fluorouracil $600 \mathrm{mg} / \mathrm{m}^{2}$, and leucovorin $20 \mathrm{mg} / \mathrm{m}^{2}$ over 4 -week cycles with all drugs given on days 1,8 and 15 . Most patients were pretreated with adjuvant and/or metastatic chemotherapy. This regimen was only moderately active, producing an overall response rate of $22 \%$ in 27 evaluable patients. Leukopenia was the predominant toxicity. Considering that gemcitabine treatment alone achieved response rates of up to $42 \%$ in pretreated patients (table 1), there is presently no indication that the addition of 5fluorouracil improves efficacy.

\section{Gemcitabine plus 5-Fluorouracil/Leucovorin and Cyclophosphamide}

In the phase I portion of a phase I/II study, gemcitabine $\left(1,000 \mathrm{mg} / \mathrm{m}^{2}\right)$ was combined with 5 -fluorouracil $\left(425 \mathrm{mg} / \mathrm{m}^{2}\right)$, folinic acid $\left(100 \mathrm{mg} / \mathrm{m}^{2}\right)$, and escalating doses of cyclophosphamide [89]. All drugs were applied on days 1 and 8 of a 3-week cycle. The subsequent phase II portion used a cyclophosphamide dose of $800 \mathrm{mg} / \mathrm{m}^{2}$ supported by G-CSF. In 21 evaluable patients refractory to anthracycline- and taxane-based chemotherapy, an overall response rate of $43 \%$ and a WHO grade $3 / 4$ neutropenia rate of $34 \%$ were reported.

\section{Gemcitabine plus a Biologic Agent: Trastuzumab}

Gemcitabine has also been combined with paclitaxel plus the biologic agent trastuzumab as first-line therapy for patients with newly diagnosed HER2/neu-overexpressing metastatic breast cancer. In preclinical studies, trastuzumab was found to have additive [90] to synergistic [91] effects with some chemotherapeutic agents in tumor cell lines. Clinical trials in HER2-positive breast cancer patients have demonstrated that trastuzumab combined with cytotoxic chemotherapy is associated with improved time to disease progression and overall survival [92].

In a first-line study conducted by the Hossier Oncology Group (HOG) [93], 27 patients (target enrollment of 46) received a triplet regimen consisting of paclitaxel $175 \mathrm{mg} /$ $\mathrm{m}^{2}$ over $3 \mathrm{~h}$ on day 1 , gemcitabine $1,200 \mathrm{mg} / \mathrm{m}^{2}$ on days 1 and 8 , and trastuzumab at a $4-\mathrm{mg} / \mathrm{kg}$ loading dose on day 1 followed by $2 \mathrm{mg} / \mathrm{kg} /$ week, every 21 days. A partial response was achieved in $92 \%$ of 13 evaluable patients, and only 1 patient has progressed. The treatment has been 
Table 6. Phase III studies planned or in progress: Gemcitabine/taxane combinations in metastatic breast cancer

\begin{tabular}{|c|c|c|c|}
\hline $\begin{array}{l}\text { Location or } \\
\text { cooperative group }\end{array}$ & Population & $\begin{array}{l}\text { Approximate } \\
\text { number }\end{array}$ & Regimens (doses in $\mathrm{mg} / \mathrm{m}^{2}$, all 21-day cycles) \\
\hline Global & $\begin{array}{l}\text { First-line } \\
\text { Prior adjuvant (anthracycline) }\end{array}$ & 500 & $\begin{array}{l}\text { Gem } 1,250 \mathrm{~d} 1 \text { plus Pac } 175 \mathrm{~d} 1 \\
\text { vs. Pac } 175 \mathrm{~d} 1\end{array}$ \\
\hline $\begin{array}{l}\text { Danish Breast Cancer } \\
\text { Cooperative Group }\end{array}$ & $\begin{array}{l}\text { First- or second-line } \\
\text { (prior anthracycline) }\end{array}$ & 300 & $\begin{array}{l}\text { Gem } 1,000 \mathrm{~d} 1,8 \text { plus Doc } 75 \mathrm{~d} 8 \\
\text { vs. Doc } 100 \mathrm{~d} 1\end{array}$ \\
\hline Finnish Cooperative Group & First-line & 240 & $\begin{array}{l}\text { Gem } 1,000 \mathrm{~d} 1,8 \text { alternating with Doc } 100 \mathrm{~d} 1 \\
\text { vs. Doc } 100 \mathrm{~d} 1\end{array}$ \\
\hline United States $^{a}$ & $\begin{array}{l}\text { First- or second-line } \\
\text { (prior anthracycline) }\end{array}$ & 440 & $\begin{array}{l}\text { Gem } 1,000 \mathrm{~d} 1,8 \text { plus Doc } 75 \mathrm{~d} 1 \\
\text { vs. Doc } 75 \mathrm{~d} 1 \text { plus Cap } 2,000 \text { or } 2,500 \mathrm{~d} 1-14\end{array}$ \\
\hline United States & $\begin{array}{l}\text { Second-line } \\
\text { (prior anthracycline) }\end{array}$ & 210 & $\begin{array}{l}\text { Gem } 1,000 \mathrm{~d} 1,8 \text { plus Doc } 75 \mathrm{~d} 1 \\
\text { vs. Gem } 1,000 \mathrm{~d} 1,8 \text { plus Vin } 25 \mathrm{~d} 1,8\end{array}$ \\
\hline Asia/Pacific & $\begin{array}{l}\text { First- or second-line } \\
\text { (prior anthracycline) }\end{array}$ & 210 & $\begin{array}{l}\text { Gem } 1,250 \mathrm{~d} 1,8 \text { plus Pac } 175 \mathrm{~d} 1 \\
\text { vs. Gem } 1,000 \mathrm{~d} 1,8 \text { plus Pac } 100 \mathrm{~d} 1,8 \\
\text { vs. Gem } 1,000 \mathrm{~d} 1,8 \text { plus Doc } 40 \mathrm{~d} 1,8\end{array}$ \\
\hline
\end{tabular}

Gem = Gemcitabine $;$ Pac $=$ paclitaxel $;$ Doc $=$ docetaxel $;$ Cap = capecitabine $;$ Vin $=$ vinorelbine.

a A similar study is also being conducted in the European Union.

well tolerated, with grade 4 toxicity limited to myelosuppression.

O'Shaughnessy et al. [94] are conducting a phase II study of gemcitabine $1,200 \mathrm{mg} / \mathrm{m}^{2}$ (days 1 and 8 ) combined with trastuzumab as a $4-\mathrm{mg} / \mathrm{kg}$ loading dose, followed by $2 \mathrm{mg} / \mathrm{kg} /$ week thereafter, in 55 heavily pretreated patients with HER2-positive metastatic breast cancer. Cycles were administered every 21 days. Among the 38 assessable patients, 12 had partial responses, for an overall response rate of $32 ; 40(6 / 15)$ and $26 \%(6 / 23)$ of responders were HER $2++$ and +++ , respectively. Median time to progression was 6.7 months. The primary grade $3 / 4$ toxicity was neutropenia, which occurred in 9 patients.

\section{Future Directions}

Due to increasing anthracycline resistance and the risk of cumulative cardiotoxicity associated with anthracylines, the development of non-anthracycline-containing regimens in the treatment of metastatic breast cancer is clearly needed. As a single agent, gemcitabine (1) is active and well tolerated even as salvage therapy, (2) can be easily combined with other agents without the confounding effects of cross-resistance and overlapping toxicity, and (3) has shown synergistic potential with various cytotox- ic as well as biologic agents. Thus, phase III trials are planned or under way to confirm the increased efficacy and manageable toxicity of gemcitabine when added to nonanthracycline agents with proven single-agent activity.

On the basis of phase II study results, combinations of gemcitabine with taxanes appear to offer the most favorable balance between efficacy and tolerability. These regimens, particularly the two-drug combinations, have demonstrated durable, high response rates and consistent median times to disease progression under various pretreatment conditions, without significantly compromising tolerability. Various phase III studies are planned or in progress to establish the role of gemcitabine in combination with a taxane as first-line or salvage therapy (table 6).

In a large randomized trial by the Central European Cooperative Oncology Group comparing the GET regimen to the triplet combination of 5-fluorouracil/epirubicin/cyclophosphamide (FEC) [95], the clinical value of the triple-agent approach is being tested as first-line chemotherapy. An interim analysis of toxicity performed on 22 patients has demonstrated that the administration of the GET regimen is unproblematic in the multicenter setting; the need for G-CSF support is rare. Neutropenia grade 3/4 was comparable in the GET and FEC arms (63 vs. $69 \%$, respectively). 
Thus far, GET and GAT trials have been performed exclusively in the first-line setting, and have yielded high response rates, perhaps at the expense of acceptable hematotoxicity. The potential of these dose-dense combinations as conditioning regimens for high-dose chemotherapy and/or stem cell support should be affirmed in phase III trials.

\section{Summary and Conclusion}

The rationale for gemcitabine-based combination treatment is supported by its unique mechanism of action, which makes drug resistance to standard pretreatment improbable, and its potential for synergistic drug interactions. Numerous trials have demonstrated activity of gemcitabine in the treatment of breast cancer. Gemcitabine has proven activity as a well-tolerated single agent and even as salvage therapy in intensively pretreated patients. It may be useful in patients who choose palliation without many side effects, particularly as its use is not precluded by cumulative organ toxicity. Its combination with cytotoxic agents such as doxorubicin, the taxanes, vinorelbine, vindesine, or cisplatin, and targeted therapy such as trastuzumab, provides feasible options with added activity.

Specifically, gemcitabine combinations provide new options in anthracycline- and taxane-pretreated patients. Gemcitabine plus taxane regimens appear to offer the most favorable balance between efficacy and tolerability. Various confirmatory phase III trials including gemcitabine/taxane combinations are planned or in progress. In addition, single-agent gemcitabine is being studied as alternating therapy with taxanes in the first-line setting and also with targeted agents.

\section{Acknowledgement}

The author gratefully acknowledges Mary Alice Miller, $\mathrm{PhD}$, for her assistance in editing and revising the manuscript.

\section{References}

1 Winer EP, Morrow M, Osborne CK, Harris JR: Malignant tumors of the breast; in De Vita VT, Hellman S, Rosenberg SA (eds): Cancer. Principles and Practice of Oncology, ed 6. Philadelphia, Lippincott Williams \& Wilkins, 2001, pp 1651-1717.

2 Chan S, Friedrichs K, Noel D, Pinter T, Van Belle S, Vorobiof D, Duarte R, Gil Gil M, Bodrogi I, Murray E, Yelle L, von Minckwitz G, Korec S, Simmonds P, Buzzi F, Gonzalez Mancha R, Richardson G, Walpole E, Ronzoni M, Murawsky M, Alakl M, Riva A: Prospective randomized trial of docetaxel versus doxorubicin in patients with metastatic breast cancer. The 303 Study Group. J Clin Oncol 1999;17: 2341-2354.

3 Paridaens R, Biganzoli L, Bruning P, Klijn JG, Gamucci T, Houston S, Coleman R, Schachter J, Van Vreckem A, Sylvester R, Awada A, Wildiers J, Piccart M: Paclitaxel versus doxorubicin as first-line single-agent chemotherapy for metastatic breast cancer: A European Organization for Research and Treatment of Cancer randomized study with cross-over. J Clin Oncol 2000;18:724-733.

4 Bishop JF, Dewar J, Toner GC, Smith J, Tattersall MH, Olver IN, Ackland S, Kennedy I, Goldstein D, Gurney H, Walpole E, Levi J, Stephenson J, Canetta R: Initial paclitaxel improves outcome compared with CMFP combination chemotherapy as front-line therapy in untreated metastatic breast cancer. J Clin Oncol 1999; 17:2355-2364.
5 Sledge GW Jr, Neuberg D, Ingle J, Martino S, Wood W: Phase III trial of doxorubicin vs. paclitaxel vs. doxorubicin + paclitaxel as firstline therapy for metastatic breast cancer: An intergroup trial. Proc Am Soc Clin Oncol 1997; 16:1a (abstr 2).

6 Barton-Burke M: Gemcitabine: A pharmacologic and clinical overview (review). Cancer Nurs 1999;22:176-183.

7 Von Hoff DD: Activity of gemcitabine in a human tumor cloning assay as a basis for clinical trials with gemcitabine. San Antonio Drug Development Team. Invest New Drugs 1996; 14:265-270.

8 Hui YF, Reitz J: Gemcitabine: A cytidine analogue active against solid tumors. Am J Health Syst Pharmacol 1997;54:162-170.

9 Heinemann V, Hertel LW, Grindey GB, Plunkett W: Comparison of the cellular pharmacokinetics and toxicity of $2^{\prime}, 2^{\prime}$-difluorodeoxycytidine and 1- $\beta-D$-arabinofuranosylcytosine. Cancer Res 1988;48:4024-4031.

10 Huang P, Chubb S, Hertel LW, Grindey GB, Plunkett W: Action of $2^{\prime}, 2^{\prime}$-difluorodeoxycytidine on DNA synthesis. Cancer Res 1991;51: 6110-6117.

11 Plunkett W, Huang P, Searcy CE, Gandhi V: Gemcitabine: Pharmacology and mechanisms of action. Semin Oncol 1996;23(suppl 10):315.

12 Green MR: Gemcitabine safety overview. Semin Oncol 1996;23(suppl 10):32-35.
13 Blackstein M, Vogel CL, Ambinder R, Covan J, Iglesias J, Melemed A: Gemcitabine as firstline therapy in patients with metastatic breast cancer: A phase II trial. Oncology 2002;62: 2-8.

14 Possinger K, Kaufmann M, Coleman R, Stuart NS, Helsing M, Ohnmacht U, Arning M: Phase II study of gemcitabine as first-line chemotherapy in patients with advanced or metastatic breast cancer. Anticancer Drugs 1999;10:155162.

15 Carmichael J, Possinger K, Phillip P, Beykirch M, Kerr H, Walling J, Harris AL: Advanced breast cancer: A phase II trial with gemcitabine. J Clin Oncol 1995;13:2731-2736.

16 Valerio MR, Cicero G, Armata MG, Bajardi E, Crosta A, Badalamenti G, Arcara C, Agosta G, Vieni S, Latteri F, Russo A, Gulotta G, Gebbia $\mathrm{N}$ : Gemcitabine in pretreated breast cancer. Proc Am Soc Clin Oncol 2001;20:51b (abstr 1953).

17 Brodowicz T, Kostler WJ, Moslinger R, Tomek S, Vaclavik I, Herscovici V, Wiltschke C, Steger GG, Wein W, Seifert M, Kubista E, Zielinski CC: Single-agent gemcitabine as secondand third-line treatment in metastatic breast cancer. Breast 2000;9:338-342.

18 Gerson R, Serrano OA, Villalobos A, Ortiz C, Sánchez-Forgach R: Gemcitabine response in advanced breast cancer in relation to immunohistochemical factors. Proc Am Soc Clin Oncol 2000;19:145a (abstr 572). 
19 Spielmann M, Llombart-Cussac A, Kalla S, Kalla S, Espié M, Namer M, Ferrero JM, Diéras V, Fumoleau P, Cuvier C, Perrocheau G, Ponzio A, Kayitalire L, Pouillart P: Singleagent gemcitabine is active in previously treated metastatic breast cancer. Oncology 2001;60:303-307.

20 Schmid P, Akrivakis K, Flath B, Grosse Y, Sezer O, Mergenthaler HG, Possinger K: Phase II trial of gemcitabine as prolonged infusion in metastatic breast cancer. Anticancer Drugs 1999; 10:625-631.

21 Smorenburg $\mathrm{CH}$, Bontenbal M, Seynaeve C, van Zuylen C, de Heus G, Jerweij J, de Wit R: Phase II study of weekly gemcitabine in patients with metastatic breast cancer relapsing or failing both an anthracycline and a taxane. Breast Cancer Res Treat 2001;66:83-87.

22 MEDLINE $^{\circledR}$ (Medical Literature, Analysis, and Retrieval System Online) database: US National Library of Medicine (accessed Oct 1 2001). Available from URL: http://www.ncbi. nlm.nih.gov/entrez/query.fcgi

23 Rahman ZU, Frye DK, Smith TL, Asmar L, Theriault RL, Buzdar AU, Hortobagyi GN: Results and long-term follow-up for $1,581 \mathrm{pa}$ tients with metastatic breast carcinoma treated with standard dose doxorubicin-containing chemotherapy: A reference. Cancer 1999;85: 104-111.

24 Perez-Manga G, Lluch A, Alba E, MorenoNogueira JA, Palomero M, Garcia-Conde J, Khayat D, Rivelles N: Gemcitabine in combination with doxorubicin in advanced breast cancer: Final results of a phase II pharmacokinetic trial. J Clin Oncol 2000;18:2545-2552.

25 Gomez H, Kahatt C, Falcon S, Santillana S, de Mendoza FH, Valdivia S, Vallejos C, Otero J Pen DL: A phase II study of neoadjuvant gemcitabine plus doxorubicin in stage IIIB breast cancer: A preliminary report. Semin Oncol 2001;28(suppl 10):57-61.

26 Luftner D, Flath B, Akrivakis C, Mergenthaler $\mathrm{HG}$, Ohnmacht U, Arning M, Possinger K: Gemcitabine plus dose-escalated epirubicin in advanced breast cancer: Results of a phase study. Invest New Drugs 1998;16:141-146.

27 Berton-Rigaud D, Viens P, Dieras V, PujadeLauraine E, Espie M, Kayitalire P, Fumoleau P, Pouillart P: Gemzar and epirubicin in patients with metastatic breast cancer: Final results of a phase I dose finding study. Proc Am Soc Clin Oncol 2000;19:122a (abstr 433).

28 Campone M, Viens P, Dieras V, Pujade-Lauraine D, Serin T, Petit T, Espie M, Kayitalire L, Robert C, Fumoleau P, Pouillart P: Gemzar (G) and epirubicin (E) in patients with metastatic breast cancer: Results of a phase II trial. Proc Am Soc Clin Oncol 2001;48b (abstr 1940).

29 Theodossiou C, Cook JA, Fisher J, Teague D, Liebmann JE, Russo A, Mitchell JB: Interaction of gemcitabine with paclitaxel and cisplatin in human tumor cell lines. Int J Oncol 1998 12:825-832.
30 Kroep JR, Tolis C, Voorn DA, Loves WJP, Van Groeningen CJ, Pinedo HM, Peters GJ: Paclitaxel and gemcitabine cell-cycle interactions in non-small-cell lung cancer cell lines. Proc Am Assoc Cancer Res 1999;40:678 (abstr 4472).

31 Jungnelius JU, Ridderheim M, Perren T, Gawande S, Roychowdhury D, Poole CJ: A phase I study of gemcitabine and paclitaxel in patients with recurrent ovarian cancer. Proc Am Soc Clin Oncol 2002;21:223a (abstr 890).

32 Fogli S, Danesi R, De Braud F, De Pas T, Curigliano G, Giovannetti G, Del Tacca M: Drug distribution and pharmacokinetic/pharmacodynamic relationship of paclitaxel and gemcitabine in patients with non-small-cell lung cancer. Ann Oncol 2001;12:1553-1559.

33 Kroep JR, Giaccone G, Voorn DA, Smit EF, Beijnen JH, Rosing $\mathrm{H}$, van Moorsel CJ, van Groeningen CJ, Postmus PE, Pinedo HM, Peters GJ: Gemcitabine and paclitaxel: Pharmacokinetic and pharmacodynamic interactions in patients with non-small-cell cancer. J Clin Oncol 1999;17:2190-2197.

34 Valero V: Docetaxel as single-agent therapy in metastatic breast cancer: Clinical efficacy. Semin Oncol 1997;24:S13-11-S13-18.

35 Cortes JH, Pazdur R: Docetaxel. J Clin Oncol 1995; 13:2643-2655.

36 Burris HA: Single-agent docetaxel (taxotere) in randomized phase III trials. Semin Oncol 1999;26(suppl 9):1-6.

37 Laufman LR, Spiridonidis CH, Pritchard, et al: Monthly docetaxel and weekly gemcitabine in metastatic breast cancer: A phase II trial. Ann Oncol 2001;12:1259-1264.

38 Kornek G, Raderer M, Fiebiger W, Scheithauer W, Haider K, Kwasny W, Depisch D, Kovats E, Lang F: Treatment of advanced breast cancer with docetaxel and gemcitabine + human granulocyte colony-stimulating factor. Proc Am Soc Clin Oncol 2001;20:57b (abstr 1978).

39 Pelegri A, Calvo L, Florian J, Mayordomo JI, Vazquez S, Srcusa A, Carrasco E, Bayo JL, Marti M, Anton A: Every-other-week combination of gemcitabine and docetaxel as first-line therapy in advanced breast cancer patients: Preliminary results from a phase II trial. Breast Cancer Res Treat 2001;69:3 (abstr 356).

40 Brandi M, Giotta F, Vici P, Valerio MR, Vinciarelli G, Gebbia V, Galetta D, Lopez M, Colucci G: Salvage chemotherapy with docetaxel and gemcitabine in metastatic breast cancer: Preliminary results of a multicenter phase II trial of GOIM (Gruppo Oncologico dell'Italia Meridionale). Proc Am Soc Clin Oncol 2001; 20:52b (abstr 1956).

41 Mavroudis D, Malamos N, Alexopoulos A, Kourousis C, Agelaki S, Sarra E, Potamianou A, Kosmas C, Rigatos G, Giannakakis T, Kalbakis K, Apostolaki F, Vlachonicolis J, Kakolyris S, Samonis G, Georgoulias V: Salvage chemotherapy in anthracycline-pretreated metastatic breast cancer patients with docetaxel and gemcitabine: A multicenter phase II trial. Greek Breast Cancer Cooperative Group. Ann Oncol 1999;10:211-215.
42 Fountzilas G, Nicolaide C, Bafaloukos D, Kalogera-Fountzila A, Kalofonos H, Samelis G, Aravantinos G, Pavlidia N et al: Docetaxel and gemcitabine in anthracycline-resistant advanced breast cancer: A Hellenic Cooperative Oncology Group phase II study. Cancer Invest 2000;18:503-509.

43 Alexopoulos A, Kandilis K, Stavrakakis J, Stavrinides H, Georganta C, Gerasimidis G, Ardavanis A, Rigatos G: Evidence for in vivo synergism between docetaxel and gemcitabine in patients with metastatic cancer. Proc Am Soc Clin Oncol 2001;20:49b (abstr 1945).

44 Holmes PA, Walters RS, Theriault RL: Phase II trial of taxol, an active drug in the treatment of metastatic breast cancer. J Natl Cancer Inst 1991;83:1797-1805.

45 Holmes FA, Valero V, Walters RS, Theriault RL, Booser DJ, Fraschini G, Buzdar AU, Frye D, Gibbs HR, Hortobagyi GN: The M.D. Anderson Cancer Center experience with taxol in metastatic breast cancer. J Natl Cancer Inst Monogr 1993;15:161-169.

46 Clemons M, Leahy M, Valle J, Jayson G, Ransom M, Howell A: Review of recent trials of chemotherapy for advanced breast cancer: The taxanes. Eur J Cancer 1997;33:2183-2193.

47 Seidman AD, Reichman BS, Crown JPA, Yao TJ, Currie V, Hakes TB, Hudis CA, Gilewski TA, Baselga J, Forsythe P, Lepore J, Marks L, Fain K, Souhrada M, Onetto N, Arbuck S, Norton L: Paclitaxel as second and subsequent therapy for metastatic breast cancer: Activity independent of prior anthracycline response. J Clin Oncol 1995;13:1152-1159.

48 Abrams JS, Vena DA, Baltz J, Adams J, Montello M, Christian M, Onetto N, DesmondHellmann S, Canetta R, Friedman MA, Arbuck SG: Paclitaxel activity in heavily pretreated breast cancer: A National Cancer Institute treatment referral center trial. J Clin Oncol 1995;13:2056-2065.

49 Nabholtz JM, Gelmon K, Bontenbal M, Spielmann M, Catimel G, Conte P, Klaassen U, Namer M, Bonneterre J, Fumoleau P, Winograd B: Multicenter, randomized comparative study of two doses of paclitaxel in patients with metastatic breast cancer. J Clin Oncol 1996;14: 1858-1867.

50 Colomer R, Llombart A, Lluch A, Ojeda B, Barnadas A, Caranana V, Fernandez Y, De Paz L, Guillem V, Alonso S: Paclitaxel/gemcitabine administered every two weeks in advanced breast cancer: Preliminary results of a phase II trial. Semin Oncol 2000;27(suppl 2):20-24.

51 Delfino C, Riva Gonzales L, Mickiewicz E, Balbiani L, Caccia G, Flores Morales D, Zori Brosio C, Comba A. Gemcitabine plus paclitaxel administered as first-line chemotherapy for patients with advances breast cancer. Proc Am Soc Clin Oncol 2002;21:53b (abstr 2025).

52 Genot JY, Tubiana-Hulin M, Tubiana-Mathiew N, Piperno-Neumann S. Gemcitabine and paclitaxel in metastatic breast cancer: A phase II study in the first-line setting. Proc Am Soc Clin Oncol 2002;21:48b (abstr 2002). 
53 Sanchez P, Medina MB, Mohedano N, Jaen A, Porras I, Gonzalez E, Fernandez M, Lozano A: Results from a phase II study of gemcitabine in combination with paclitaxel in metastatic breast cancer. Ann Oncol 1998 (abstr 77P).

54 Murad AM, Guimaraes RC, Aragao BC, Scalabrini-Neto AO, Rodrigues VH, Garcia R: Phase II trial of the use of paclitaxel and gemcitabine as a salvage treatment in metastatic breast cancer. Am J Clin Oncol 2001;24:264268.

55 Gregory RK, Smith IE: Vinorelbine - A clinical review. Br J Cancer 2000;82:1907-1913.

56 Jones S, Winer E, Vogel C, Laufman L, Hutchins L, O'Rourke M, Lembersky B, Budman D, Bigley J, Hohneker J: Randomized comparison of vinorelbine and melphalan in anthracycline-refractory advanced breast cancer. J Clin Oncol 1995;13:2567-2574.

57 Degardin M, Bonneterre J, Hecquet B, Pion JM, Adenis A, Horner D, Demaille A: Vinorelbine (navelbine) as a salvage treatment for advanced breast cancer. Ann Oncol 1994;5: 423-426.

58 Haider K, Kornek GV, Kwasny W, Weinlander G, Valencak J, Lang F, Puribauer F, Kovats E, Depisch D, Scheithauer W: Treatment of advanced breast cancer with gemcitabine and vinorelbine plus human granulocyte colonystimulating factor. Breast Cancer Res Treat 1999;55:203-211.

59 Valenza R, Leonardi V, Gebbia V, Agostara B: Gemcitabine and vinorelbine in pretreated advanced breast cancer: A pilot study. Ann Oncol 2000;11:495-496

60 Stathopoulos GP, Rigotos SK, Pergantas N, Tsavdarides D, Athanasiodia I, Malamos NA, Stothopoulos JG: Phase II trial of biweekly administration of vinorelbine and gemcitabine in pretreated advanced breast cancer. J Clin Oncol 2002;20:37-41

61 Nicolaides C, Dimopoulos MA, Samantas E, Bafaloukos D, Kalofonos C, Fountzilas G, Razi E, Kosmidis P, Pavlidis N: Gemcitabine and vinorelbine as second-line treatment in patients with metastatic breast cancer progressing after first-line taxane-based chemotherapy: A phase II study conducted by the Hellenic Cooperative Oncology Group. Ann Oncol 2000;11: 873-875.

62 Mariani G, Tagliabue P, Zucchinelli P, Brambilla C, Demicheli R, Villa E, Marchiano A, Valagussa P, Bonadonna G, Gianni L: Phase I/II study of gemcitabine in association with vinorelbine for metastatic breast cancer. Breast Cancer Res Treat 2001;70:163-169.

63 Donadio M, Ritoro G, Novarino A, Bonazzi G, Corrorullo Z, Buffani L, Occelli M, Berteno O. Phase II study of gemcitabine and vinorelbine in metastatic breast cancer. Ann Oncol 2000; 11(suppl 4):31 (abstr 128p).

64 Moser R, Hausmaninger H, Mlineritsch B, Steger G, Haidinger R, Thaler J, Samonigg H: Gemcitabine and vinorelbine as first- or second-line therapy in patients with advanced breast cancer. A prospective phase II trial. Proc Am Soc Clin Oncol 2001;20:56b (abstr 1973).

Gemcitabine in Advanced and Metastatic Breast Cancer
65 Gokmen E, Karabulut B, Sezgin C, Sanal S: A phase II study of gemcitabine and vinorelbine in patients with advanced breast cancer. Proc Am Soc Clin Oncol 2000;19:110a (abstr 427).

66 Cazzaniga ME, Poletti P, Ardizzoia A, Bollina R, Bernardo G, Bonciarelli G, Pancera G, Labianca R, Malugani F, Barni S: A new alternative for second-line chemotherapy in advanced breast cancer patients: Gemcitabine-vindesine. A Giscad study. Proc Am Soc Clin Oncol 2001; 20:50b (abstr 1946).

67 Yap HY, Salem P, Hortobogyi GN, Bodey GP Sr, Buzdar AU, Tashima CK, Blumenschein GR: Phase II study of cis-dichlorodiamminedichloroplatinum(II) in advanced breast cancer. Cancer Treat Rep 1978;62:405-408.

68 Ostrow S, Egorin M, Aisner J, Bachur N, Wiernik PH: High-dose cis-diamminedichloroplatinum therapy in patients with advanced breast cancer. Pharmacokinetics, toxicity and therapeutic efficacy. Cancer Clin Trials 1980;3:2327.

69 Forastiere AA, Hakes TB, Wittes JT, Wittes RE: Cisplatin in the treatment of metastatic breast carcinoma: A prospective randomized trial of two dosage schedules. Am J Clin Oncol 1982;5:243-247.

70 Bull T, Anderson ME, Lippman JG, et al: A phase II trial of cis-dichlorodiammine platinum II in breast and ovarian carcinomas. Proc Am Soc Clin Oncol 1978;19:87 (abstr 345).

71 Samal B, Vaitheviclus V, Singlekowenia A: Cisdiamminedichloroplatinum (CDDP) in advanced breast and colorectal carcinoma. Proc Am Soc Clin Oncol 1978;19:347 (abstr 164).

72 Peters GJ, Bergman AM, Ruiz van Haperen VW, Veerman G, Kuiper CM, Braakhuis BJ: Interaction between cisplatin and gemcitabine in vitro and in vivo. Semin Oncol 1995;22 (suppl 11):72-79.

73 Pegram MD, Lipton A, Hayes DF, Weber BL, Baselga JM, Tripathy D, Baly D, Baughman SA, Twaddell T, Glaspy JA, Slamon DJ: Phase II study of receptor-enhanced chemosensitivity using recombinant humanized antip185HER2/neu monoclonal antibody plus cisplatin in patients with HER2/neu-overexpressing metastatic breast cancer refractory to chemotherapy treatment. J Clin Oncol 1998;16: 2659-2671.

74 Nagourney RA, Su YZ, Link J, DiSaia P, Evans SS: Gemcitabine synergy with cisplatin. Clinical and laboratory correlates. Proc Am Assoc Cancer Res 1998;39:310 (abstr 2118).

75 Calderillo Ruíz GC, Fuentes de la Peña H, Meza FA, Aguirre G, Ramírez M, Avila E: A phase II study of gemcitabine plus cisplatin in metastatic breast cancer. Proc Am Soc Clin Oncol 2001;20:60b (abstr 1988).

76 Nagourney RA, Link JS, Blitzer JB, Forsthoff C, Evans SS: Gemcitabine plus cisplatin repeating doublet therapy in previously treated, relapsed breast cancer patients. J Clin Oncol 2000; 18:2245-2249.

77 Chaudhry S, Abdel-Rahman HA, Patil R, Mansour R, Mills G, Burton GV: Prospective phase II study of weekly cisplatin-gemcitabine in refractory metastatic breast cancer. Proc Am Soc Clin Oncol 2000;19:111a (abstr 430).
78 Burch PA, Mailliard JA, Hillman DW, Perez EA, Krook JE, Rowland KM, Ingle JN: Phase II study of gemcitabine and cisplatin in patients with metastatic breast cancer and failure on prior chemotherapy: A North Central Cancer Treatment Group trial. Breast Cancer Res Treat 2000;64:81 (abstr 322).

79 Doroshow JH, Tetef M, Margolin K, Somlo G, Frankel P, Longmate J, Synold T, Gandara D, Lenz HJ, Albain KS: Significant activity of gemcitabine and cisplatin in both heavily and minimally pretreated metastatic breast cancer patients: A California Cancer Consortium/ Loyola University Chicago Trial. Proc Am Soc Clin Oncol 2000; 19:155a (abstr 609H).

80 Galvez CA, Galmarini F, Curie M: Monthly cisplatin and gemcitabine as second-line chemotherapy for patients with advanced breast cancer. Breast Cancer Res Treat 2000;64:81 (abstr 324).

81 Evans S, Chow C, Su YZ, Hilton R, Horlick D, Harris C, Nagourney R: Is cisplatin and gemcitabine equivalent to carboplatin and gemcitabine? Yes and no. Proc Am Soc Clin Oncol 2001;20:99b (abstr 2145)

82 Conte PF, Gennari A, Donati S, Salvadori B, Baldini E, Bengala C, Pazzagli I, Orlandini C, Danesi R, Fogli S, Del Tucca M: Gemcitabine plus epirubicin plus taxol in advanced breast cancer: A phase II study. Breast Cancer Res Treat 2001;68:171-179.

83 Mazzoni F, Donati S, Gennari A, Cetto G, Crino L, Galligioni E, Lucenti A, Mansutti M, Molino AM, Tumolo S, Conte PF: A multicentric phase II trial of gemcitabine plus epirubicin plus taxol (GET) as first-line chemotherapy for metastatic breast cancer. Breast Cancer Res Treat 2000;64:87 (abstr 347).

84 Sanchez-Rovira P, Jaen A, Gonzalez E, Porras I, Duenas R, Medina B, Mohedano N, Fernandez M, Martos M, Lozano A, Carrasco E: Biweekly gemcitabine, doxorubicin and paclitaxel as first-line treatment in metastatic breast cancer: Final results from a phase II trial. Oncology 2001;15(suppl 3):44-47.

85 Sanchez-Rovira P, Ramirez C, Duenas R, Medina B, Porras I, Cozar A, Fernandez M, Mohedano N, Lozano A: HER2 status as a predictive factor for pathological complete response in primary breast cancer patients treated with gemcitabine-adriamycin-taxol as neoadjuvant treatment. Breast Cancer Res Treat 2001;69: 246.

86 Sanchez-Rovira P, Jaen A, Duenas R, Porras I, Carrasco E, Duenas B, Medina B, Fernandez M, Mohedano N, Martinez-Muro JL, PerezGracia JL, Lozano A: Primary chemotherapy in locally advanced breast cancer with gemcitabine, adriamycin and paclitaxel. A phase II trial and predictive value of ERB2. Proc Am Soc Clin Oncol 2001;20:31b (abstr 1872).

87 Schulz L, Schalhorn A, Wilmanns W, Heinemann V: Synergistic interaction of gemcitabine and 5-fluorouracil in colon cancer cells. Proc Am Soc Clin Oncol 1998; 17:251a (abstr 965). 
88 Mulkerin D, Dietrich L, Koch P, Douglas J, Fass T, Richie K, Stewart J: A phase II study of gemcitabine, 5-fluorouracil, and leucovorin in women with metastatic breast cancer. Proc Am Soc Clin Oncol 2001;20:43b (abstr 1919).

89 Carreca I, Frasci G, D’Aiuto G, Comella P, Thomas R, Capasso I, Cortino GR, De Rosa V, Comella G: Gemcitabine-cyclophosphamidefluorouracil/folinic acid triplet combination in anthracycline- and taxane-refractory breast cancer patients: A SICOG phase I-II study. Proc Am Soc Clin Oncol 2001;20:54b (abstr 1963).

90 Pegram MD, Lopez A, Konecny G, Slamon DJ: Trastuzumab and chemotherapeutics: Drug interactions and synergies. Semin Oncol 2000; 27(suppl 11):21-25.
91 Hirsch FR, Helfrich B, Franklin WA, VarellaGarcia M, Chan DC, Bunn PA Jr: Preclinical studies of gemcitabine and trastuzumab in breast and lung cancer cell lines. Clin Breast Cancer 2002;3(suppl):12-16.

92 Slamon DJ, Leyland-Jones B, Shak S, Fuchs H, Paton V, Bajamonde A, Fleming T, Eiermann W, Wolter J, Pegram M, Baselga J, Norton L: Use of chemotherapy plus a monoclonal antibody against HER2 for metastatic breast cancer that overexpresses HER2. N Engl J Med 2000;344:783-792.

93 Miller KD, Sisk J, Ansari R, Gize G, Nattam S, Pennington K, Manaco F, Sledge GW Jr: Gemcitabine, paclitaxel and trastuzumab in metastatic breast cancer. Oncology 2001;15(suppl 3):38-40.
94 O’Shaugnessy JA, Vukelja S, Marsland T, Kimmel G, Ratnam S, Pippen J: Gemcitabine and trastuzumab for HER-2-positive metastatic breast cancer: Preliminary results of a phase II study. Breast Cancer Res Treat 2001;69:302.

95 Zielinski C, Beslija S, Cervek J, Mrsic-Krmpotic Z, Tchernozemsky I, Wiltschke C, Ghilezan N, Grgic M, Jassem J, Tzekova V, Kahan Z, Pawlega J, Onat $\mathrm{H}$, Inbar M, Brodowicz $\mathrm{T}$ Gemcitabine/epirubicin/paclitaxel vs. 5-fluorouracil/epirubicin/cyclophosphamide as firstline treatment in metastatic breast cancer: Interim toxicity analysis of a randomised, multicenter phase III trial of the Central European Cooperative Oncology Group (CECOG). Proc Am Soc Clin Oncol 2001;20:53b (abstr 1958). 